\title{
Infinite Volume Limit for Correlation functions in the Dipole Gas
}

\author{
Tuan Minh Le * \\ Department of Mathematics \\ SUNY at Buffalo \\ Buffalo, NY 14260
}

August 19, 2018

\begin{abstract}
We study a classical lattice dipole gas with low activity in dimension $d \geq 3$. We investigate long distance properties by a renormalization group analysis. We prove that various correlation functions have an infinite volume limit. We also get estimates on the decay of correlation functions.
\end{abstract}

\section{Introduction}

\subsection{Overview}

In this paper we continue to study the classical dipole gas on a unit lattice $\mathbb{Z}^{d}$ with $d \geq 3$. Each dipole is described by its position coordinate $x \in \mathbb{Z}^{d}$ and a unit polarization vector (moment) $p \in \mathbb{S}^{d-1}$.

Let $\left\{e_{1}, \ldots, e_{d}\right\}$ be the standard basic for $\mathbb{Z}^{d}$. For $\varphi: \mathbb{Z}^{d} \rightarrow \mathbb{R}$ and $\mu \in\{1, \ldots, d\}$ we define $\partial_{\mu} \varphi$ as $\partial_{\mu} \varphi(x)=\varphi\left(x+e_{\mu}\right)-\varphi(x)$. Let $e_{-\mu}=-e_{\mu}$ with $\mu \in\{1, \ldots, d\}$. Then the definition of $\partial_{\mu} \varphi$ can be used to define the forward or backward lattice derivative along the unit vector $e_{\mu}$ with $\mu \in\{ \pm 1, \ldots, \pm d\}$. We have that $\partial_{\mu}$ and $\partial_{-\mu}$ are adjoint to each other and $-\Delta=1 / 2 \sum_{ \pm \mu=1}^{d} \partial_{\mu}^{*} \partial_{\mu}=1 / 2 \sum_{ \pm \mu=1}^{d} \partial_{-\mu} \partial_{\mu} 1$

As in [5], the potential energy between unit dipoles $\left(x, p_{1}\right)$ and $\left(y, p_{2}\right)$ is

$$
\left(p_{1} \cdot \partial\right)\left(p_{2} \cdot \partial\right) C(x-y)
$$

where $x, y \in \mathbb{Z}^{d}$ are positions, $p_{1}, p_{2} \in \mathbb{S}^{d-1}$ are moments, $\partial=\left(\partial_{1}, \ldots, \partial_{d}\right)$ and $C(x-y)$ is the Coulomb potential on the unit lattice $\mathbb{Z}^{d}$, which is the kernel of the inverse Laplacian

$$
C(x, y)=(-\Delta)^{-1}(x, y)=(2 \pi)^{-d} \int_{[-\pi, \pi]^{d}} \frac{e^{i p \cdot(x-y)}}{2 \sum_{\mu=1}^{d}\left(1-\cos p_{\mu}\right)} d p
$$

And the potential energy of $n$ dipoles, including self energy, has the form

$$
\sum_{1 \leq k, j \leq n}\left(p_{k} \cdot \partial\right)\left(p_{j} \cdot \partial\right) C\left(x_{k}, x_{j}\right)
$$

Let $\Lambda_{N}$ be a box in $\mathbb{R}^{d}$

$$
\Lambda_{N}=\left[\frac{-L^{N}}{2}, \frac{L^{N}}{2}\right]^{d}
$$

\footnotetext{
*e-mail addresses: tuanle@buffalo.edu_or leminhtuan912@gmail.com

${ }^{1}$ We distinguish forward and backward derivatives to facilitate a symmetric decomposition of $V\left(\Lambda_{N}\right)$ (defined in (9)) into blocks.
} 
where $L \geq 2^{d+3}+1$ is a very large, odd integer. For $\Lambda_{N} \cap \mathbb{Z}^{d}$, the classical statistical mechanics of a gas of such dipoles with inverse temperature (for convenience) $\beta=1$ and activity (fugacity) $z>0$ is given by the grand canonical partition function

$$
Z_{N}=\sum_{n \geq 0} \frac{z^{n}}{n !} \prod_{i=1}^{n} \sum_{x_{i} \in \mathbb{Z}^{d} \cap \Lambda_{N}} \int_{\mathbb{S}^{d-1}} d p_{i} \exp \left(\frac{-1}{2} \sum_{1 \leq k, j \leq n}\left(p_{k} \cdot \partial\right)\left(p_{j} \cdot \partial\right) C\left(x_{k}, x_{j}\right)\right)
$$

All fundamental objects to study, such as the pressure, truncated correlation functions, etc. are derived from $Z_{N}$ and similar objects.

The model can be equivalently expressed as a Euclidean field theory (due to Kac [13] and Siegert [16]) and is given by

$$
\begin{aligned}
{ }_{0} Z_{N} \equiv Z_{N} & =\int \exp \left(z W\left(\Lambda_{N}, \phi\right)\right) d \mu_{C}(\phi) \\
\text { where } \quad W\left(\Lambda_{N}, \phi\right) & =\sum_{x \in \Lambda_{N} \cap \mathbb{Z}^{d}} \int_{\mathbb{S}^{d-1}} d p \cos (p \cdot \partial \phi(x))
\end{aligned}
$$

with

- $d p$ : the standard normalized rotation invariant measure on $\mathbb{S}^{d-1}$.

- The fields $\phi(x)$ : a family of Gaussian random variables (on some abstract measure space) indexed by $x \in \mathbb{Z}^{d}$ with mean zero and covariance $C(x, y)$ which is a positive definite function as given above.

- The measure $\mu_{C}$ : the underlying measure (see section 11.2 [6] and Appendix A [5] for more detail). We discuss about the equivalence of (5) and (6) in appendix $\mathrm{A}$ of this paper.

For investigating the truncated correlation functions, we consider a more general version of (6):

$$
{ }_{f} Z_{N}=\int \exp \left(i f(\phi)+z W\left(\Lambda_{N}, \phi\right)\right) d \mu_{C}(\phi)
$$

where $f(\phi)$ can be:

1. $f(\phi)=0$ as in [5].

2. $f(\phi)=\sum_{k=1}^{m} t_{k} \partial_{\mu_{k}} \phi\left(x_{k}\right)$. We use this $f(\phi)$ to study the truncated correlation functions $\mathcal{G}^{t}\left(x_{1}, x_{2}, \ldots x_{m}\right) \equiv\left\langle\prod_{k=1}^{m} \partial_{\mu_{k}} \phi\left(x_{k}\right)\right\rangle^{t}=\left.i^{m} \frac{\partial^{m}}{\partial t_{1} \ldots \partial t_{m}} \log { }_{f} Z^{\prime}\right|_{t_{1}=0, \ldots t_{m}=0}$ which is nontrivial and previously investigated by Dimock and Hurd [7].

3. $f(\phi)=\sum_{k=1}^{m} t_{k} \exp \left(i \partial_{\mu_{k}} \phi\left(x_{k}\right)\right)$ (for studying the dipole correlation $\left.\left\langle\prod_{k=1}^{m} \exp \left(i \partial_{\mu_{k}} \phi\left(x_{k}\right)\right)\right\rangle^{t}=\left.i^{m} \frac{\partial^{m}}{\partial t_{1} \ldots \partial t_{m}} \log { }_{f} Z^{\prime}\right|_{t_{1}=0, \ldots t_{m}=0}\right)$.

4. Other general form which will be discussed at the end of this paper. The general form can be applied for truncated correlations of density of the dipoles which also has been studied by Brydges and Keller [2]. We think that this general form has more applications.

Here $x_{k} \in \mathbb{Z}^{d}$ are different points; $\mu_{k} \in\{ \pm 1, \ldots, \pm d\}$ and $t_{k}$ small and complex; $m \geq 2$. For the set $\left\{x_{1}, \ldots, x_{m}\right\} \subset \mathbb{Z}^{d}$, let $\operatorname{diam}\left(x_{1}, \ldots, x_{m}\right)=\max _{1 \leq i, j \leq m} \operatorname{dist}\left(x_{i}, x_{j}\right)$ where $\operatorname{dist}\left(x_{i}, x_{j}\right)$ is the distance between $x_{i}$ and $x_{j}$ on lattice $\mathbb{Z}^{d}$.

To get rid of the boundary and study the long distance properties of the system, we would like to take the thermodynamic limit for these quantities, i.e. the limit as $N \rightarrow \infty$ which is so called infinite 
volume limit. Actually $Z_{N}$ is not expected to have a limit as $N \rightarrow \infty$. In [5], Dimock has established an infinite volume limit for the pressure defined by

$$
p_{N}=\left|\Lambda_{N}\right|^{-1} \log _{0} Z_{N}
$$

(with $|z|$ sufficiently small). Such infinite volume limits have also been obtained by Frohlich and Park [10] and by Frohlich and Spencer [11. They used a method of correlation inequalities.

In this paper, we continue the study of the long distance properties of the dipole gas model. For long distance (i.e., when $|x-y|$ large), the potential $\partial_{\mu} \partial_{\nu} C(x-y)$ behaves like $\mathcal{O}\left(|x-y|^{-d}\right)$, that means it is not integrable and we could not use the theory of the Mayer expansion to establish such results. To overcome this problem, we use the method of the renormalization group.

We follow particularly a Renormalization Group approach recently developed by Brydges and Slade [1] and Dimock [5. We generalize Dimock's framework with an external field and obtain some estimates on the correlation functions as in Dimock and Hurd [7, and Brydges and Keller [2]. The main result is the existence of the infinite volume limit for correlations functions, which is new. Earlier work using RG approach to the dipole gas can be found in Gawedski and Kupiainen [12, Brydges and Yau [4.

Besides the dipole gas papers mentioned above, we would like to cite some other papers on the Coulomb gas in $d=2$ which has a dipole phase. There are the works of Dimock and Hurd [8], Falco 9] and Zhao [17.

\subsection{The main result}

For our RG approach we follow the analysis of Brydges' lecture [1]. Instead of (7), we use a different finite volume approximation. First, we add an extra term $(1-\varepsilon) V\left(\Lambda_{N}, \phi\right)$ where $0<\varepsilon$ is closed to 1 and

$$
V\left(\Lambda_{N}, \phi\right)=\frac{1}{4} \sum_{x \in \Lambda_{N} \cap \mathbb{Z}^{d}} \sum_{ \pm \mu=1}^{d}\left(\partial_{\mu} \phi(x)\right)^{2}
$$

By replacing the covariance $C$ by $\varepsilon^{-1} C$, this extra term will be partially compensated. Hence instead of (7) we will consider a new finite volume generating function

$$
{ }_{f} Z_{N}={ }_{f} Z_{N}^{\prime} / Z_{N}^{\prime \prime}
$$

where

$$
{ }_{f} Z_{N}^{\prime}=\int e^{i f(\phi)} \exp \left(z W\left(\Lambda_{N}, \phi\right)-(1-\varepsilon) V\left(\Lambda_{N}, \phi\right)\right) d \mu_{\varepsilon^{-1} C}(\phi)
$$

and

$$
Z_{N}^{\prime \prime}=\int \exp \left(-(1-\varepsilon) V\left(\Lambda_{N}, \phi\right)\right) d \mu_{\varepsilon^{-1} C}(\phi)
$$

We have

$$
\begin{aligned}
{ }_{f} Z_{N} & ={ }_{f} Z_{N}^{\prime} / Z_{N}^{\prime \prime} \\
& =\int e^{i f(\phi)} \exp \left(z W\left(\Lambda_{N}, \phi\right)\right)\left[\left(Z{ }_{N}\right)^{-1} \exp \left(-(1-\varepsilon) V\left(\Lambda_{N}, \phi\right)\right) d \mu_{\varepsilon^{-1} C}(\phi)\right]
\end{aligned}
$$

When $N \rightarrow \infty, \exp \left(-(1-\varepsilon) V\left(\Lambda_{N}, \phi\right)\right)$ formally becomes $\exp (1 / 2(1-\varepsilon)(\phi,-\Delta \phi))$, and $d \mu_{\varepsilon^{-1} C}(\phi)=$ $(1 / 2(\varepsilon)(\phi,-\Delta \phi)) d \phi$. So the bracketed expression formally converges to (const.) $(1 / 2(\phi,-\Delta \phi)) d \phi=$ (const.) $d \mu_{C}(\phi)$ when $N \rightarrow \infty$. Formally this new ${ }_{f} Z_{N}$ gives the same limit as (7). This result holds for any choice of $\varepsilon$. By definition (9), the extra term $(1-\varepsilon) V\left(\Lambda_{N}, \phi\right)=(1-\varepsilon) \frac{1}{4} \sum_{x \in \Lambda_{N} \cap \mathbb{Z}^{d}} \sum_{ \pm \mu=1}^{d}\left(\partial_{\mu} \phi(x)\right)^{2}$. 
Therefore the choice of $\varepsilon$ is a choice of how much $(\partial \phi)^{2}$ one is putting in the interaction and how much in the measure .

Similarly to the Theorem 1 in [5], our main theorems are:

Theorem 1 For $|z|$ and $\max _{k}\left|t_{k}\right|$ sufficiently small there is an $\varepsilon=\varepsilon(z)$ close to 1 so that ${ }_{f} p_{N}=\left|\Lambda_{N}\right|^{-1} \log \left({ }_{f} Z_{N}\right)$ has a limit as $N \rightarrow \infty 2$

Using $f(\phi)=\sum_{k=1}^{m} t_{k} \partial_{\mu_{k}} \phi\left(x_{k}\right)$, we achieve some estimate for the correlation functions:

Theorem 2 For any small $\epsilon>0$, with $L, A$ sufficiently large (depending on $\epsilon$ ), $\eta=\min \{d / 2,2\}$, we have:

$$
\left|\left\langle\prod_{k=1}^{m} \partial_{\mu_{k}} \phi\left(x_{k}\right)\right\rangle^{t}\right| \leq \frac{m !}{a^{m}} \operatorname{diam}^{-\eta+\epsilon}\left(x_{1}, \ldots x_{m}\right)
$$

where a depends on $\epsilon, L, A$.

And we also can obtain the existence of infinite volume limit for correlation functions.

Theorem 3 With L, A sufficiently large, the infinite volume limit of truncated correlation function

$$
\lim _{N \rightarrow \infty}\left\langle\prod_{k=1}^{m} \partial_{\mu_{k}} \phi\left(x_{k}\right)\right\rangle^{t} \text { exists }
$$

When $d=3$ or 4 , the result in Theorem 2 looks like the result in 7 , but here it is obtained with the new method.

Using $f(\phi)=\sum_{k=1}^{m} t_{k} \exp \left(i \partial_{\mu_{k}} \phi\left(x_{k}\right)\right)$, we can obtain Theorems 10 and 11 which are similar to Theorems 2 and 3 , just with different $f$.

At the end of this paper, we investigate a general form of $f$ and obtain Theorems 12 and 13 . Applying theorem [12 with a special $f$ for density of dipoles, we have obtained some estimates for truncated correlation functions of density of dipoles with $(m \geq 2)$ points (Corollary 1) instead of only 2 points as Theorem 1.1.2 in 2]. Then we apply theorem 13 to establish the infinite volume limit for truncated correlation functions of density of dipoles (Corollary 21).

For the proof of Theorem 11 we will show that, with a suitable choice of $\varepsilon=\varepsilon(z)$, the density $\exp (z W-(1-\varepsilon) V)$ likely goes to zero under the renormalization group flow and leaves a measure like $\mu_{\varepsilon(z)^{-1} C}$ to describe the long distance behavior of the system. Accordingly $\varepsilon(z)$ can be interpreted as a dielectric constant.

Now we rewrite the generating function ${ }_{f} Z_{N}$. First we scale $\phi \rightarrow \phi / \sqrt{\varepsilon}$ and then let $\sigma=\varepsilon^{-1}-1$. Because $\varepsilon$ is closed to 1 , we have $\sigma$ is near zero. We also have

$$
\begin{aligned}
{ }_{f} Z_{N}^{\prime}(z, \sigma) & =\int e^{i f(\phi)} \exp \left(z W\left(\Lambda_{N}, \sqrt{1+\sigma} \phi\right)-\sigma V\left(\Lambda_{N}, \phi\right)\right) d \mu_{C}(\phi) \\
Z_{N}^{\prime \prime}(\sigma) & \left.=\int \exp \left(-\sigma V\left(\Lambda_{N}, \phi\right)\right)\right) d \mu_{C}(\phi) \\
{ }_{f} Z_{N}(z, \sigma) & ={ }_{f} Z_{N}^{\prime}(z, \sigma) / Z_{N}^{\prime \prime}(\sigma)
\end{aligned}
$$

Then we need to show that with $|z|$ sufficiently small there is a (smooth) $\sigma=\sigma(z)$ near zero such that,

$$
\left|\Lambda_{N}\right|^{-1} \log _{f} Z_{N}(z, \sigma(z))=\left|\Lambda_{N}\right|^{-1} \log _{f} Z_{N}^{\prime}(z, \sigma(z))-\left|\Lambda_{N}\right|^{-1} \log Z_{N}^{\prime \prime}(\sigma(z))
$$

has a limit when $N \rightarrow \infty$. And theorem 1 is proved just by putting $\varepsilon(z)=(1+\sigma(z))^{-1}$ back. Dimock has proved that, for small real $\sigma$ with $|\sigma|<1$, we have $\left|\Lambda_{N}\right|^{-1} \log Z_{N}^{\prime \prime}(\sigma)$ converges as $N \rightarrow \infty$ (Theorem 2, [5]). Hence we only need to investigate the first term in (16)

The paper is organized as follows:

\footnotetext{
${ }^{2}$ In Theorem $1 f(\phi)$ can be $0, \sum_{k=1}^{m} t_{k} \partial_{\mu_{k}} \phi\left(x_{k}\right)$, or $\sum_{k=1}^{m} t_{k} \exp \left(i \partial_{\mu_{k}} \phi\left(x_{k}\right)\right)$.
} 
- In section 2 we give some general definitions on the lattice and its properties. We also give definitions about the norms we use together with their crucial properties and estimates. Then we define the basic Renormalization Group transformation as in ([5]).

- In section 3 we accomplish the detailed analysis of the Renormalization Group transformation to isolate the leading terms. Then we simplify them for the next scale.

- In section 8 we study the RG flow and find the stable manifold $\sigma=\sigma(z)$.

- In section 5 we assemble the results and prove the infinite volume limit for $\left|\Lambda_{N}\right|^{-1} \log _{f} Z_{N}^{\prime}$ exists.

- Finally in section 6 , by combining all the other estimates, we obtain some estimates for correlation functions and establish the infinite volume limit of correlation functions.

\section{Preliminaries}

In this section, we quote all notations and basic result from Dimock $[\underline{5}$. At the same time, we introduce some new notations which are useful for this paper.

\subsection{Multiscale decomposition}

RG methods are based upon a multiscale decomposition of the basic lattice covariance $C$ into a sequence of more controllable integrals and analyze the effects separately at each stage. Especially we choose a decomposition into finite range covariances which is developed by Brydges, Guadagni, and Mitter [3]. The decomposition of the lattice covariance $C$ has the form

$$
C(x-y)=\sum_{j=1}^{\infty} \Gamma_{j}(x-y)
$$

such that

- $\Gamma_{j}(x)$ is defined on $\mathbb{Z}^{d}$, is positive semi-definite, and satisfies the finite range property: $\Gamma_{j}(x)=0$ if $|x| \geq L^{j} / 2$.

- There is a constant $c_{0}$ independent of $L$ such that, for all $j, x$, we have

$$
\left|\Gamma_{j}(x)\right| \leq c_{0} L^{-(j-1)(d-2)}
$$

This implies that the series converges uniformly.

- There are constants $c_{\alpha}$ independent of $L$ such that

$$
\left|\partial^{\alpha} \Gamma_{j}(x)\right| \leq c_{\alpha} L^{-(j-1)(d-2+|\alpha|)}
$$

where $\partial^{\alpha}=\prod_{ \pm \mu=1}^{d} \partial_{\mu}^{\alpha_{\mu}}$ is a multi-derivative and $|\alpha|=\sum_{\mu}\left|\alpha_{\mu}\right|$. Thus the differentiated series converges uniformly to $\partial^{\alpha} C$.

- (Lemma 2, [5]) There are some constants $C_{L, \alpha}$ such that

$$
\left|\partial^{\alpha} C(x)\right| \leq C_{L, \alpha}(1+|x|)^{-d+2-|\alpha|}
$$

For our RG analysis we need to break off pieces of covariance $C(x-y)$ one at a time. So we define

$$
C_{k}(x-y)=\sum_{j=k+1}^{\infty} \Gamma_{j}(x-y)
$$

Hence we have $C=C_{0}$ and

$$
C_{k}(x-y)=C_{k+1}(x-y)+\Gamma_{k+1}(x-y)
$$




\subsection{Renormalization Group Transformation}

The generating function (15) can be rewritten as

$$
{ }_{f} Z_{N}^{\prime}(z, \sigma)=\int{ }_{f} \mathcal{Z}_{0}^{N}(\phi) d \mu_{C_{0}}(\phi)
$$

with

$$
{ }_{f} \mathcal{Z}_{0}^{N}(\phi)=e^{i f(\phi)} \exp \left(z W\left(\Lambda_{N}, \sqrt{1+\sigma} \phi\right)-\sigma V\left(\Lambda_{N}, \phi\right)\right)
$$

We use the left subscript $f$ as an extra notation for 3 cases at the same time:

- $f(\phi)=0$ as in (Dimock, [5]);

- $\left.f(\phi)=\sum_{k=1}^{m} t_{k} \partial_{\mu_{k}} \phi\left(x_{k}\right)\right)$;

- $f(\phi)=\sum_{k=1}^{m} t_{k} \exp \left(i \partial_{\mu_{k}} \phi\left(x_{k}\right)\right)$.

Since $C_{0}=C_{1}+\Gamma_{1}$ we replace an integral over $\mu_{C_{0}}$ by an integral over $\mu_{\Gamma_{1}}$ and $\mu_{C_{1}}$. So we have

$$
{ }_{f} Z_{N}^{\prime}(z, \sigma)=\int{ }_{f} \mathcal{Z}_{0}^{N}(\phi+\zeta) d \mu_{\Gamma_{1}}(\zeta) d \mu_{C_{1}}(\phi)=\int{ }_{f} \mathcal{Z}_{1}^{N}(\phi) d \mu_{C_{1}}(\phi)
$$

We define a new density by the fluctuation integral

$$
{ }_{f} \mathcal{Z}_{1}^{N}(\phi)=\left(\mu_{\Gamma_{1}}{ }_{f} \mathcal{Z}_{0}^{N}\right)(\phi) \equiv \int{ }_{f} \mathcal{Z}_{0}^{N}(\phi+\zeta) d \mu_{\Gamma_{1}}(\zeta)
$$

Because $\Gamma_{1}, C_{1}$ are only positive semi-definite, these are degenerate Gaussian measures 3 By continuing this way, we will have the representation for $j=0,1,2, \ldots$

$$
{ }_{f} Z_{N}^{\prime}(z, \sigma)=\int{ }_{f} \mathcal{Z}_{j}^{N}(\phi) d \mu_{C_{j}}(\phi)
$$

here the density ${ }_{f} \mathcal{Z}_{j}^{N}(\phi)$ is defined by

$$
{ }_{f} \mathcal{Z}_{j+1}^{N}(\phi)=\left(\mu_{\Gamma_{j+1}} *{ }_{f} \mathcal{Z}_{j}^{N}\right)(\phi)=\int{ }_{f} \mathcal{Z}_{j}^{N}(\phi+\zeta) d \mu_{\Gamma_{j+1}}(\zeta)
$$

Our job is to investigate the growth of these densities when $j$ go to $\infty$.

\subsection{Local expansion}

We will rewrite each density ${ }_{f} \mathcal{Z}_{j}^{N}(\phi)$ in a form which presents its locality properties known as a polymer representation. The localization becomes coarser when $j$ gets bigger. First we will give some basic definitions on the lattice $\mathbb{Z}^{d}$.

\subsubsection{Basic definitions on the lattice $\mathbb{Z}^{d}$}

For $j=0,1,2, \ldots$ we partition $\mathbb{Z}^{d}$ into $j$-blocks $B$. These blocks have side $L^{j}$ and are translates of the center $j$-blocks

$$
B_{j}^{0}=\left\{x \in \mathbb{Z}^{d}:|x|<1 / 2\left(L^{j}-1\right)\right\}
$$

by points in the lattice $L^{j} \mathbb{Z}^{d}$. The set of all $j$-blocks in $\Lambda=\Lambda_{N}$ is denoted $\mathcal{B}_{j}\left(\Lambda_{N}\right), \mathcal{B}_{j}(\Lambda)$ or just $\mathcal{B}_{j}$. A union of $j$-blocks $X$ is called a $j$-polymer. Note that $\Lambda$ is also a $j$-polymer for $0 \leq j \leq N$. The set of

\footnotetext{
${ }^{3}$ Dimock has discussed these in Appendix A, [5].
} 
all $j$-polymers in $\Lambda=\Lambda_{N}$ is denoted $\mathcal{P}_{j}(\Lambda)$ or just $\mathcal{P}_{j}$. The set of all connected $j$-polymers is denoted by $\mathcal{P}_{j, c}$. Let $X \in \mathcal{P}_{j}$, the closure $\bar{X}$ is the smallest $Y \in \mathcal{P}_{j+1}$ such that $X \subset Y$.

For a $j$-polymer $X$, let $|X|_{j}$ be the number of $j$-blocks in $X$. We call $j$-polymer $X$ a small set if it is connected and contains no more than $2^{d} j$-blocks. The set of all small set $j$-polymers in $\Lambda$ is denoted by $\mathcal{S}_{j}(\Lambda)$ or just $\mathcal{S}_{j}$. A $j$-block $B$ has a small set neighborhood $B^{*}=\cup\left\{Y \in \mathcal{S}_{j}: Y \supset B\right\}$. Note: If $B_{1}, B_{2}$ are $j$-blocks and $B_{2} \in B_{1}^{*}$ then, using above definition, we also have that $B_{1} \in B_{2}^{*}$. Similarly a $j$-polymer $X$ has a small set neighborhood $X^{*}$.

For $l \geq 1$ and integer $d$, we define some constants $n_{1}(d), n_{2}(d), n_{3}(d, l)$ which are bounded and, for every $j \geq 0$, we have:

$$
\begin{aligned}
n_{1}(d) & \equiv \sum_{X \in \mathcal{S}_{0}, X \supset 0} 1 /|X|_{0}=\sum_{X \in \mathcal{S}_{j}, X \supset B_{j}^{0}} 1 /|X|_{j} \\
n_{2}(d) & \equiv \sum_{X \in \mathcal{S}_{0}, X \supset 0} 1=\sum_{X \in \mathcal{S}_{j}, X \supset B_{j}^{0}} 1 \\
n_{3}(d, l) & \equiv \sum_{X \in \mathcal{S}_{0}, X \supset 0} \frac{l^{-|X|_{0}}}{|X|_{0}}=\sum_{X \in \mathcal{S}_{j}, X \supset B_{j}^{0}} \frac{l^{-|X|_{j}}}{|X|_{j}} \\
n_{3}(d, l) & \leq n_{3}(d, 1)=n_{1}(d) \leq n_{2}(d) \leq\left(2^{d}\right) !(2 d)^{2^{d}}
\end{aligned}
$$

Furthermore, with a fixed $d$, we can get

$$
0 \leq \lim _{l \rightarrow \infty} n_{3}(d, l) \leq \lim _{l \rightarrow \infty} \frac{n_{1}(d)}{l}=0
$$

\subsubsection{Local expansion}

Using the same approach as in [5], we rewrite the density ${ }_{f} \mathcal{Z}_{j}^{N}(\phi)$ for $\phi: \mathbb{Z}^{d} \rightarrow \mathbb{R}$ in the the general form

$$
{ }_{f} \mathcal{Z}=\left({ }_{f} I \circ{ }_{f} K\right)(\Lambda) \equiv \sum_{X \in \mathcal{P}_{j}(\Lambda)}{ }_{f} I(\Lambda-X)_{f} K(X)
$$

Here ${ }_{f} I(Y)$ is a background functional which is explicitly known and carries the main contribution to the density. The ${ }_{f} K(X)$ is so called a polymer activity. It represents small corrections to the background.

In section $\left[5\right.$ we will show that the initial density ${ }_{f} I_{0}$ has the factor property. We want to keep this factor property at all scales. Then we can use the analysis of Brydges' lecture [1. Therefore we assume ${ }_{f} I(Y)$ always is in the form of

$$
{ }_{f} I(Y)=\prod_{B \in \mathcal{B}_{j}: B \subset Y}{ }_{f} I(B)
$$

and ${ }_{f} I(B, \phi)$ depends on $\phi$ only $B^{*}$, the small set neighborhood of $B$. Moreover we assume ${ }_{f} K(X)$ factors over the connected components $\mathcal{C}(X)$ of $X$

$$
{ }_{f} K(X)=\prod_{Y \subset \mathcal{C}(X)}{ }_{f} K(Y)
$$

and that ${ }_{f} K(X, \phi)$ only depends on $\phi$ in $X^{*}$.

As in [5], the background functional ${ }_{f} I(B)$ has a special form: ${ }_{f} I\left({ }_{f} E, \sigma, B\right)=\exp \left(-V\left({ }_{f} E, \sigma, B\right)\right)$ where 4

$$
V\left({ }_{f} E, \sigma, B, \phi\right)={ }_{f} E(B)+\frac{1}{4} \sum_{x \in B} \sum_{\mu \nu} \sigma_{\mu \nu}(B) \partial_{\mu} \phi(x) \partial_{\nu} \phi(x)
$$

\footnotetext{
${ }^{4}$ Sums over $\mu$ are understood to range over $\mu= \pm 1, \ldots, \pm d$, unless otherwise specified.
} 
for some functions ${ }_{f} E, \sigma_{\mu \nu}: \mathcal{B}_{j} \rightarrow \mathbb{R}$. Indeed we usually can take $\sigma_{\mu \nu}(B)=\sigma \delta_{\mu \nu}$ for some constant $\sigma$. Then $V\left({ }_{f} E, \sigma, B, \phi\right)$ becomes

$$
V\left({ }_{f} E, \sigma, B, \phi\right)={ }_{f} E(B)+\frac{\sigma}{4} \sum_{x \in B} \sum_{\mu}\left(\partial_{\mu} \phi(x)\right)^{2} \equiv{ }_{f} E(B)+\sigma V(B)
$$

Also in our model, when $f=0$, we will have

$$
{ }_{0} K(X, \phi)={ }_{0} K(X,-\phi) \quad{ }_{0} K(X, \phi)={ }_{0} K(X, \phi+c)
$$

The later holds for any constant $c$ which means that ${ }_{0} K(X, \phi)$ only depends on derivatives $\partial \phi$.

\subsection{About norms and their properties}

In this paper we use exactly the same norms and notations as in Dimock [5]. Now we consider potential $V(s, B, \phi)$ of the form

$$
V(s, B, \phi)=\frac{1}{4} \sum_{x \in B} \sum_{\mu \nu} s_{\mu \nu}(x) \partial_{\mu} \phi(x) \partial_{\nu} \phi(x)
$$

here the norms of functions $s_{\mu \nu}(x)$ are defined by

$$
\|s\|_{j}=\sup _{B \in \mathcal{B}_{j}}|B|^{-1}\|s\|_{1, B}=\sup _{B \in \mathcal{B}_{j}} L^{-d j} \sum_{\mu \nu} \sum_{x \in B}\left|s_{\mu \nu}(x)\right|
$$

If $s_{\mu \nu}(x)=\sigma \delta_{\mu \nu}$ then $V(s, B)=\sigma V(B)$ as defined in (36) and the norm $\|s\|_{j}=2 d \sigma$.

The following lemmas are some results from Section 3 in [5]:

Lemma 1 (Lemma 3, [5])

1. For any functional $s_{\mu \nu}(x)$, we have

$$
\begin{aligned}
\|V(s, B)\|_{s, j}^{\prime} & \leq h^{2}\|s\|_{j} \\
\|V(s, B)\|_{s, j} & \leq h^{2}\|s\|_{j}
\end{aligned}
$$

2. The function $\sigma \rightarrow \exp (-\sigma V(B))$ is complex analytic and if $h^{2} \sigma$ is sufficiently small, we have

$$
\begin{aligned}
\left\|e^{-\sigma V(B)}\right\|_{s, j}^{\prime} & \leq 2 \\
\| e^{-\sigma V(B)} & \|_{s, j} \leq 2
\end{aligned}
$$

Let $c$ be a constant such that the function $\sigma \rightarrow \exp (-\sigma V(B))$ is analytic in $|\sigma| \leq c h^{-2}$ and satisfies $\|\exp (-\sigma V(B))\|_{s, j} \leq 2$ on that domain.

To start the RG transformation, we also need some estimate on the initial interaction. When $j=0$, $B \in \mathcal{B}_{0}$ is just a single site $x \in \mathbb{Z}^{d}$, so we consider

$$
W(u, B, \phi)=\int_{\mathbb{S}^{d-1}} d p \cos (p \cdot \partial \phi(x) u)
$$

Lemma 2 (Lemma 4, [5])

1. $W(u, B)$ is bounded by

$$
\|W(u, B)\|_{s, 0} \leq 2 e^{\sqrt{d} h u}
$$

We also have that $W(u, B)$ is strongly continuously differentiable in $u$.

2. $e^{z W(u, B)}$ is complex analytic in $z$ and satisfies, for $|z|$ sufficiently small (depending on $d, h, u$ ), we have

$$
\left\|e^{z W(u, B)}\right\|_{s, 0} \leq 2
$$

And $e^{z W(u, B)}$ is also strongly continuously differentiable in $u$. 


\section{Analysis of the RG Transformation}

Now we use the Brydges-Slade RG analysis and follow the framework of Dimock [5], but with an external field $f$.

\subsection{Coordinates $\left({ }_{f} I_{j},{ }_{f} K_{j}\right)$}

Continuing to the subsection 2.3.2 (Local Expansion), we suppose that we have ${ }_{f} \mathcal{Z}(\phi)=\left({ }_{f} I{ }_{f} K\right)(\Lambda, \phi)$ with polymers on scale $j$. We rewrite it as

$$
{ }_{f} \mathcal{Z}^{\prime}\left(\phi^{\prime}\right)=\left(\mu_{\Gamma_{j+1}} *{ }_{f} \mathcal{Z}\right)\left(\phi^{\prime}\right) \equiv \int{ }_{f} \mathcal{Z}\left(\phi^{\prime}+\zeta\right) d \mu_{\Gamma_{j+1}}(\zeta)
$$

here we try to put it back to the form

$$
{ }_{f} \mathcal{Z}^{\prime}\left(\phi^{\prime}\right)=\left({ }_{f}^{\prime} I^{\prime}{ }_{f} K^{\prime}\right)\left(\Lambda, \phi^{\prime}\right)
$$

where the polymers are now on scale $(j+1)$. Furthermore, supposed that we have chosen ${ }_{f} I^{\prime}$, we will find ${ }_{f} K^{\prime}$ so the identity holds. As explained before, our choice of ${ }_{f} I^{\prime}$ is to have the form

$$
{ }_{f} I^{\prime}\left(B^{\prime}, \phi^{\prime}\right)=\prod_{B \in \mathcal{B}_{j}, B \subset B^{\prime}} \tilde{f} I\left(B, \phi^{\prime}\right) \quad B^{\prime} \in \mathcal{B}_{j+1}
$$

Now we define

$$
\begin{aligned}
\delta_{f} I\left(B, \phi^{\prime}, \zeta\right) & ={ }_{f} I\left(B, \phi^{\prime}+\zeta\right)-\tilde{{ }_{f} I} I\left(B, \phi^{\prime}\right) \\
{ }_{f} K \circ \delta_{f} I \equiv{ }_{f} K\left(X, \phi^{\prime}, \zeta\right) & =\sum_{Y \subset X}{ }_{f} K\left(Y, \phi^{\prime}+\zeta\right) \delta_{f} I^{X-Y}\left(\phi^{\prime}, \zeta\right)
\end{aligned}
$$

For connected $X$ we write ${ }_{f} \tilde{K}\left(X, \phi^{\prime}, \zeta\right)$ in the form 5

$$
{ }_{f} \tilde{K}\left(X, \phi^{\prime}, \zeta\right)=\sum_{B \subset X}{ }_{f} J\left(B, X, \phi^{\prime}\right)+{ }_{f} K\left(X, \phi^{\prime}, \zeta\right)
$$

Given ${ }_{f} K$ and ${ }_{f} J$ the equation (49) would give us a definition of ${ }_{f} \breve{K}(X)$ for $X$ connected. And for any $X \in \mathcal{P}_{j}$, we define

$$
{ }_{f} \check{K}\left(X, \phi^{\prime}, \zeta\right)=\prod_{Y \in \mathcal{C}(X)}{ }_{f} \check{K}\left(Y, \phi^{\prime}, \zeta\right)
$$

After using the finite range property and making some rearrangements as Proposition 5.1, Brydges [1], we have (46) holds with

$$
{ }_{f} K^{\prime}\left(U, \phi^{\prime}\right)=\sum_{X, \chi \rightarrow U}{ }_{f} J^{\chi}\left(\phi^{\prime}\right)_{f} I^{U-\left(X_{\chi} \cup X\right)}\left(\phi^{\prime}\right)_{f} \check{K}^{\#}\left(X, \phi^{\prime}\right) \quad U \in \mathcal{P}_{j+1}
$$

where $\chi=\left(B_{1}, X_{1}, \ldots B_{n}, X_{n}\right)$ and the condition $X, \chi \rightarrow U$ means that $X_{1}, \ldots X_{n}, X$ be strictly disjoint and satisfy $\overline{\left(B_{1}^{*} \cup \cdots \cup B_{n}^{*} \cup X\right)}=U$. Moreover

$$
\begin{aligned}
& { }_{f} J^{\chi}\left(\phi^{\prime}\right)=\prod_{i=1}^{n}{ }_{f} J\left(B_{l}, X_{l}, \phi^{\prime}\right) \\
& \tilde{f}^{U-\left(X_{\chi} \cup X\right)}\left(\phi^{\prime}\right)=\prod_{B \in U-\left(X_{\chi} \cup X\right)} \tilde{f} I\left(B, \phi^{\prime}\right)
\end{aligned}
$$

\footnotetext{
${ }^{5}$ As in (Dimock, [5] $),{ }_{f} J(B, X)$ will be chosen to depend on ${ }_{f} K$ and required ${ }_{f} J(B, X)=0$ unless $X \in \mathcal{S}_{j}, B \subset X$ and that ${ }_{f} J\left(B, X, \phi^{\prime}\right)$ depend on $\phi^{\prime}$ only in $B^{*}$.
} 
with $X_{\chi}=\cup_{i} X_{i}$. And ${ }_{f} K^{\#}\left(X, \phi^{\prime}\right)$ is ${ }_{f} \check{K}\left(X, \phi^{\prime}, \zeta\right)$ integrated over $\zeta$ as (78) in [5].

At this point ${ }_{f} K^{\prime}$ is considered as a function of ${ }_{f} I,{ }_{f} I,{ }_{f} J,{ }_{f} K$. It vanishes at the point $\left({ }_{f} I,{ }_{f} I,{ }_{f} J,{ }_{f} K\right)=$ $(1,1,0,0)$ since $\chi=\emptyset$ and $X=\emptyset$ iff $U=\emptyset$. We study its behavior in a neighborhood of this point. We have the norm on ${ }_{f} K$ as $(75)$ in $[5]$ and we define

$$
\begin{aligned}
\left\|_{f} I\right\|_{s, j} & =\sup _{B \in \mathcal{B}_{j}}\left\|_{f} I(B)\right\|_{s, j} \\
\left\|_{f} I\right\|_{s, j}^{\prime} & =\sup _{B \in \mathcal{B}_{j}}\left\|_{f} I(B)\right\|_{s, j}^{\prime} \\
\left\|_{f} J\right\|_{j}^{\prime} & =\sup _{X \in \mathcal{S}_{j}, B \subset X}\left\|_{f} J(X, B)\right\|_{j}^{\prime}
\end{aligned}
$$

We also set

$$
\delta_{f} K={ }_{f} K-{ }_{0} K
$$

Using the same argument as Theorem 3 in [5], we have the following result.

Theorem 4 Let $A$ be sufficiently large.

1. For $R>0$ there is a $r>0$ such that the following holds for all $j$. If $\left\|_{f} I-1\right\|_{s, j}<r,\left\|{ }_{f} I-1\right\|_{s, j}^{\prime}<$ $r, \max \left\{\left\|_{f} J\right\|_{j}^{\prime},\left\|_{0} J\right\|_{j}^{\prime}\right\}<r$ and $\max \left\{\left\|_{f} K\right\|_{j},\left\|_{0} K\right\|_{j}\right\}<r$ then $\max \left\{\left\|_{f} K^{\prime}\right\|_{j+1},\left\|_{0} K^{\prime}\right\|_{j+1}\right\}<R$. Furthermore ${ }_{f} K^{\prime}$ is a smooth function of ${ }_{f} I,{ }_{f} I,{ }_{f} J,{ }_{f} K$ on this domain with derivatives bounded uniformly in $j$. The analyticity of ${ }_{f} K^{\prime}$ in $t_{1}, \ldots, t_{m}$ still holds when we go from $j$-scale to $(j+1)$-scale.

2. If also

$$
\sum_{X \in \mathcal{S}_{j}: X \supset B} f(B, X)=0
$$

then the linearization of ${ }_{f} K^{\prime}={ }_{f} K^{\prime}\left({ }_{f} I, \tilde{f} I,{ }_{f} J,{ }_{f} K\right)$ at $\left({ }_{f} I, \tilde{f} I,{ }_{f} J,{ }_{f} K\right)=(1,1,0,0)$ is

$$
\sum_{\substack{X \in \mathcal{P}_{j, c} \\ \bar{X}=U}}\left({ }_{f} K^{\#}(X)+\left({ }_{f} I^{\#}(X)-1\right) 1_{X \in \mathcal{B}_{j}}-(\tilde{f} I(X)-1) 1_{X \in \mathcal{B}_{j}}-\sum_{B \subset X}{ }_{f} J(B, X)\right)
$$

where

$$
{ }_{f} K^{\#}(X, \phi)=\int{ }_{f} K(X, \phi+\zeta) d \mu_{\Gamma_{j+1}}(\zeta)
$$

and $_{0} J$ actually is ${ }_{f} J$ at $f=0$.

\subsection{Choosing $J$ and Estimating $\mathcal{L}_{1}, \mathcal{L}_{2}$}

\subsubsection{Choosing $J$}

For a smooth function $g(\phi)$ on $\phi \in \mathbb{R}^{\Lambda}$ let $T_{2} g$ denote a second order Taylor expansion:

$$
\begin{aligned}
& \left(T_{2} g\right)(\phi)=g(0)+g_{1}(0 ; \phi)+\frac{1}{2} g_{2}(0 ; \phi, \phi) \\
& \left(T_{0} g\right)(\phi)=g(0)
\end{aligned}
$$

With ${ }_{f} K^{\#}$ defined in (57), for $X \in \mathcal{S}_{j}, X \supset B, X \neq B$, we pick:

$$
\begin{aligned}
{ }_{f} J(B, X) & =\frac{1}{|X|_{j}}\left[T_{2}\left({ }_{0} K^{\#}(X)\right)+T_{0}\left({ }_{f} K^{\#}(X)\right)-T_{0}\left({ }_{0} K^{\#}(X)\right)\right] \\
& =\frac{1}{|X|_{j}}\left[T_{2}\left({ }_{0} K^{\#}(X)\right)+T_{0}\left(\delta_{f} K^{\#}(X)\right)\right]
\end{aligned}
$$


and choose ${ }_{f} J(B, B)$ so that (55) is satisfied. Otherwise, we let ${ }_{f} J(B, X)=0$.

As in (36) we have picked

$$
{ }_{f} I(B)={ }_{f} I\left({ }_{f} E, \sigma, B\right)=\exp \left(-V\left({ }_{f} E, \sigma, B\right)\right)
$$

So we require ${ }_{f} I$ to have the same form

$$
{ }_{f} I(B)={ }_{f} I\left({ }_{f} E, \tilde{\sigma}, B\right)=\exp \left(-V\left({ }_{f} E, \tilde{\sigma}, B\right)\right)
$$

with ${ }_{f} E, \tilde{\sigma}$ which will be defined later. Because $\sum_{B \subset B^{\prime}} V(B)=V\left(B^{\prime}\right)$, we have

$$
{ }_{f} I^{\prime}\left(B^{\prime}\right)={ }_{f} I\left({ }_{f} E^{\prime}, \sigma^{\prime}, B^{\prime}\right)=\exp \left(-V\left({ }_{f} E^{\prime}, \sigma^{\prime}, B\right)\right)
$$

with

$$
\begin{aligned}
{ }_{f} E^{\prime}\left(B^{\prime}\right) & =\sum_{B \subset B^{\prime}} \tilde{f} \tilde{E}(B) \\
\sigma^{\prime} & =\tilde{\sigma}
\end{aligned}
$$

The map ${ }_{f} K^{\prime}$ becomes ${ }_{f} K^{\prime}={ }_{f} K^{\prime}\left({ }_{f} E, \tilde{\sigma},{ }_{f} E, \sigma,{ }_{f} K,{ }_{0} K\right)$. We use the standard norm on the energy

$$
\left\|_{f} E\right\|_{j}=\sup _{B \in \mathcal{B}_{j}}\left|{ }_{f} E(B)\right|
$$

And the theorem 4 becomes:

Theorem 5 Let $A$ be sufficiently large.

1. For $R>0$ there is a $r>0$ such that the following holds for all $j$. If $\left\|_{f} \tilde{E}\right\|_{j},|\tilde{\sigma}|,\|\|_{f} E \|_{j},|\sigma|$, $\max \left\{\left\|_{f} K\right\|_{j},\left\|_{0} K\right\|_{j}\right\}<r$ then $\max \left\{\left\|_{f} K^{\prime}\right\|_{j+1},\left\|_{0} K^{\prime}\right\|_{j+1}\right\}<R$. Furthermore ${ }_{f} K^{\prime}$ is a smooth function of ${ }_{f} E, \tilde{\sigma},{ }_{f} E, \sigma,{ }_{f} K,{ }_{0} K$ on this domain with derivatives bounded uniformly in $j$. The analyticity of ${ }_{f} K^{\prime}$ in $t_{1}, \ldots, t_{m}$ still holds when we go from $j$-scale to $(j+1)$-scale.

2. The linearization of ${ }_{f} K^{\prime}$ at the origin has the form

$$
\mathcal{L}_{1}\left({ }_{f} K\right)+\mathcal{L}_{2}\left({ }_{f} K\right)+\mathcal{L}_{3}\left({ }_{f} E, \sigma,{ }_{f} E, \tilde{\sigma},{ }_{f} K,{ }_{0} K\right)
$$

where

$$
\begin{aligned}
\mathcal{L}_{1}\left({ }_{f} K\right)(U)= & \sum_{\substack{X \in \mathcal{P}_{j, c}: \\
X \notin \mathcal{S}_{j}, \bar{X}=U}}{ }_{f} K^{\#}(X) \\
= & \sum_{\substack{X \in \mathcal{P}_{j, c}: \\
X \notin \mathcal{S}_{j}, \bar{X}=U}}{ }_{0} K^{\#}(X)+\sum_{\substack{X \in \mathcal{P}_{j, c}: \\
X \notin \mathcal{S}_{j}, \bar{X}=U}} \delta_{f} K^{\#}(X) \\
= & \mathcal{L}_{1}\left({ }_{0} K\right)(U)+\mathcal{L}_{1}\left(\delta_{f} K\right)(U) \\
\mathcal{L}_{2}\left({ }_{f} K\right)(U)= & \sum_{X \in \mathcal{S}_{j}}{ }_{f} K^{\#}(X)-\left[T_{2}\left({ }_{0} K^{\#}(X)\right)+T_{0}\left(\delta_{f} K^{\#}(X)\right)\right] \\
= & \sum_{X \in \mathcal{S}_{j}}\left(I-T_{2}\right)\left({ }_{0} K^{\#}(X)\right)+\sum_{X \in \mathcal{S}_{j}}\left(I-T_{0}\right)\left(\delta_{f} K^{\#}(X)\right) \\
= & \mathcal{L}_{2}\left({ }_{0} K\right)(U)+\mathcal{L}_{2}\left(\delta_{f} K\right)(U) \\
\mathcal{L}_{3}(E, \sigma, E, E & \left.\tilde{\sigma},{ }_{f} K,{ }_{0} K\right)(U)=\sum_{\bar{B}=U}\left(V(\tilde{E}, \tilde{\sigma}, B)-V^{\#}(E, \sigma, B)\right) \\
& +\sum_{\bar{B}=U} \sum_{X \in \mathcal{S}_{j}} \frac{1}{|X|_{j}}\left[T_{2}\left({ }_{0} K^{\#}(X)\right)+T_{0}\left(\delta_{f} K^{\#}(X)\right)\right]
\end{aligned}
$$


Proof. The new map actually is the composition of the map ${ }_{f} K^{\prime}={ }_{f} K^{\prime}\left({ }_{f} I,{ }_{f} I,{ }_{f} J,{ }_{f} K\right)$ of theorem 4 with the maps ${ }_{f} I={ }_{f} I\left({ }_{f} E, \sigma\right),{ }_{f} I={ }_{f} I\left({ }_{f} \tilde{E}, \tilde{\sigma}\right),{ }_{f} J={ }_{f} J\left({ }_{f} K,{ }_{0} K\right)$. Thus it suffices to establish uniform bounds and smoothness for the latter.

In the case $f=0$, we've already have the proof in Theorem 4, Dimock [5. So we only consider the case $f \neq 0$.

For ${ }_{f} I={ }_{f} I\left({ }_{f} E, \sigma\right), \tilde{f} I={ }_{f} I\left({ }_{f} E, \tilde{\sigma}\right)$ the proof is the same as the proof for $I, I^{\prime}$ in (Theorem 4, [5]). We have:

$$
\begin{aligned}
{ }_{f} J(B, X) & =\frac{1}{|X|_{j}}\left[T_{2}\left({ }_{0} K^{\#}(X)\right)+T_{0}\left({ }_{f} K^{\#}(X)\right)-T_{0}\left({ }_{0} K^{\#}(X)\right)\right] \\
& =\frac{1}{|X|_{j}}\left[\left(T_{2}-T_{0}\right)\left({ }_{0} K^{\#}(X)\right)+T_{0}\left({ }_{f} K^{\#}(X)\right)\right]
\end{aligned}
$$

With the same argument as in (Theorem 4, 5]), we obtain:

$$
\begin{aligned}
\left\|\left(T_{2}-T_{0}\right)\left({ }_{0} K^{\#}(X)\right)\right\|_{j}^{\prime} & \leq \mathcal{O}(1)\left\|_{0} K^{\#}(X)\right\|_{j}^{\prime} \\
\left\|\left(T_{0}\right)\left({ }_{f} K^{\#}(X)\right)\right\|_{j}^{\prime} & \leq \mathcal{O}(1)\left\|_{f} K^{\#}(X)\right\|_{j}^{\prime}
\end{aligned}
$$

By (79) in [5] these are bounded by $\mathcal{O}(1)\left[\left\|_{f} K\right\|_{j}+\left\|_{0} K\right\|_{j}\right]$. The same bound holds for $\left\|_{f} J(B, B)\right\|_{j}^{\prime}$. Therefore $\left\|_{f} J\right\|_{j}^{\prime} \leq \mathcal{O}(1)\left[\left\|_{f} K\right\|_{j}+\left\|_{0} K\right\|_{j}\right]$.

The linearization is just a computation. Indeed ${ }_{f} J(B, X)$ is designed so that

$$
\begin{aligned}
\sum_{X \in \mathcal{S}_{j}, \bar{X}=U} & \left({ }_{f} K^{\#}(X)-\sum_{B \subset X}{ }_{f} J(B, X)\right) \\
= & \sum_{\bar{B}=U} \sum_{X \in \mathcal{S}_{j}, X \supset B} \frac{1}{|X|_{j}}\left[T_{2}\left({ }_{0} K^{\#}(X)\right)+T_{0}\left(\delta_{f} K^{\#}(X)\right)\right] \\
& +\sum_{X \in \mathcal{S}_{j}, \bar{X}=U}{ }_{f}^{\#}(X)-\left[T_{2}\left({ }_{0} K^{\#}(X)\right)+T_{0}\left(\delta_{f} K^{\#}(X)\right)\right]
\end{aligned}
$$

which accounts for the presence of these terms. Also the linearization of $\left({ }_{f} I^{\#}(B)-1\right)$ is $-V^{\#}\left({ }_{f} E, \sigma, B\right)$, and so forth. This completes the proof.

\subsubsection{Estimating $\mathcal{L}_{1}, \mathcal{L}_{2}$ - the first two linearization parts}

Next we make some estimates on the linearization's parts. First we estimate $\mathcal{L}_{1}$ which is the linearization on the large $j$-polymers

Lemma 3 Let $A$ be sufficiently large depending on $L$. Then the operator $\mathcal{L}_{1}$ is a contraction with a norm which goes to zero as $A \rightarrow \infty$.

Proof. We use the same proof as in (Dimock, [5], Lemma 5), but with updated notations. We estimate by $(79)$ and $(80)$ in 5

$$
\begin{aligned}
\left\|\mathcal{L}_{1}\left({ }_{0} K\right)(U)\right\|_{j+1} \leq\left\|\mathcal{L}_{1}\left({ }_{0} K\right)(U)\right\|_{j}^{\prime} & \leq \sum_{X \notin \mathcal{S}_{j}, \bar{X}=U}\left\|_{0} K^{\#}(X)\right\|_{j}^{\prime} \\
& \leq \sum_{X \notin \mathcal{S}_{j}, \bar{X}=U}(A / 2)^{-|X|_{j}}\left\|_{0} K\right\|_{j}
\end{aligned}
$$


and

$$
\begin{aligned}
\left\|\mathcal{L}_{1}\left(\delta_{f} K\right)(U)\right\|_{j+1} \leq\left\|\mathcal{L}_{1}\left(\delta_{f} K\right)(U)\right\|_{j}^{\prime} & \leq \sum_{X \notin \mathcal{S}_{j}, \bar{X}=U}\left\|\delta_{f} K^{\#}(X)\right\|_{j}^{\prime} \\
& \leq \sum_{X \notin \mathcal{S}_{j}, \bar{X}=U}(A / 2)^{-|X|_{j}}\left\|\delta_{f} K\right\|_{j}
\end{aligned}
$$

Multiplying by $A^{|U|_{j+1}}$ then taking the supremum over $U$, these yield

$$
\begin{aligned}
\left\|\mathcal{L}_{1}\left({ }_{0} K\right)\right\|_{j+1} & \leq\left[\sup _{U} A^{|U|_{j+1}} \sum_{X \notin \mathcal{S}, \bar{X}=U}(A / 2)^{-|X|_{j}}\right]\left\|_{0} K\right\|_{j} \\
\left\|\mathcal{L}_{1}\left(\delta_{f} K\right)\right\|_{j+1} & \leq\left[\sup _{U} A^{|U|_{j+1}} \sum_{X \notin \mathcal{S}, \bar{X}=U}(A / 2)^{-|X|_{j}}\right]\left\|\delta_{f} K\right\|_{j}
\end{aligned}
$$

Using lemma 6.18 [1] the bracketed expression goes to zero as $A \rightarrow \infty$. And we also have

$$
\begin{aligned}
\left\|\mathcal{L}_{1}\left({ }_{f} K\right)\right\|_{j+1} & \leq\left\|\mathcal{L}_{1}\left({ }_{0} K\right)\right\|_{j+1}+\left\|\mathcal{L}_{1}\left(\delta_{f} K\right)\right\|_{j+1} \\
\left\|\delta_{f} K\right\|_{j} & \leq\left\|_{0} K\right\|_{j}+\left\|_{f} K\right\|_{j}
\end{aligned}
$$

Hence for $A$ sufficiently large $\left\|\mathcal{L}_{1}\left({ }_{f} K\right)\right\|_{j+1}$ is arbitrarily small. The idea of lemma 6.18 [1 is that, for large polymers $X$ such that $\bar{X}=U$, the quantity $|X|_{j}$ must be much larger than $|U|_{j+1}$.

(Q.E.D)

Now we estimate and find an explicit upper bound for $\mathcal{L}_{2}$

Lemma 4 Let $L$ be sufficiently large. Then the operator $\mathcal{L}_{2}$ is a contraction with a norm which goes to zero as $L \rightarrow \infty$.

Proof. For $f=0$, we have the Lemma 6 , in 5 .

For $f \neq 0$, we can write

$$
\begin{aligned}
\mathcal{L}_{2}\left({ }_{f} K\right)(U) & =\sum_{X \in \mathcal{S}_{j}, \bar{X}=U}\left\{\left(I-T_{2}\right)_{0} K^{\#}(X)+\left(I-T_{0}\right) \delta_{f} K^{\#}(X)\right\} \\
& =\mathcal{L}_{2}\left({ }_{0} K\right)(U)+\mathcal{L}_{2}\left(\delta_{f} K\right)(U)
\end{aligned}
$$

where

$$
\mathcal{L}_{2}\left(\delta_{f} K\right)(U)=\sum_{X \in \mathcal{S}_{j}, \bar{X}=U}\left(I-T_{0}\right) \delta_{f} K^{\#}(X)
$$

Using $([1,(6.40))$ as well as $([5]$, Lemma 6$)$ we get:

$$
\begin{aligned}
\left\|\left(I-T_{2}\right)_{0} K^{\#}(X, \phi)\right\|_{j+1} & \leq\left(1+\|\phi\|_{\Phi_{j+1}\left(X^{*}\right)}\right)^{3}\left\|_{0} K_{3}^{\#}(X, \phi)\right\|_{j+1} \\
& \leq 4\left(1+\|\phi\|_{\Phi_{j+1}\left(X^{*}\right)}^{3}\right)\left\|_{0} K_{3}^{\#}(X, \phi)\right\|_{j+1} \\
\left\|\left(I-T_{0}\right) \delta_{f} K^{\#}(X, \phi)\right\|_{j+1} & \leq\left(1+\|\phi\|_{\Phi_{j+1}\left(X^{*}\right)}\right)\left\|\delta_{f} K_{1}^{\#}(X, \phi)\right\|_{j+1}
\end{aligned}
$$

Notice that $\delta_{f} K^{\#}(X, 0)={ }_{f} K^{\#}(X, 0)-{ }_{0} K^{\#}(X, 0)$ only depend on $\phi$ in $X^{*}$. Moreover, ${ }_{0} K$ and ${ }_{f} K$ are different only on $\operatorname{supp}(f)=\left\{x_{1}, \ldots, x_{m}\right\}$. So, if $X^{*} \cap\left\{x_{1}, x_{2}, \ldots x_{m}\right\}=\emptyset$ then ${ }_{f} K^{\#}(X, 0)={ }_{0} K^{\#}(X, 0)$ which means $\delta_{f} K^{\#}(X, 0)=0$. Therefore $\delta_{f} K^{\#}(X, 0)=0$ unless $X^{*} \cap\left\{x_{1}, x_{2}, \ldots x_{m}\right\} \neq \emptyset$ 
Using property (64) in [5], we have

$$
\begin{aligned}
\left\|_{0} K_{3}^{\#}(X, \phi)\right\|_{j+1} & \leq L^{-3 d / 2}\left\|_{0} K_{3}^{\#}(X, \phi)\right\|_{j} \\
& \leq 6\left(L^{-3 d / 2}\right)\left\|_{0} K^{\#}(X, \phi)\right\|_{j} \\
& \leq 6\left(L^{-3 d / 2}\right)\left\|_{0} K^{\#}(X)\right\|_{j}^{\prime} G_{j}(X, \phi, 0) \\
\left\|\delta_{f} K_{1}^{\#}(X, \phi)\right\|_{j+1} & \leq L^{-d / 2}\left\|\delta_{f} K_{1}^{\#}(X, \phi)\right\|_{j} \\
& \leq L^{-d / 2}\left\|\delta_{f} K^{\#}(X, \phi)\right\|_{j} \\
& \leq L^{-d / 2}\left(\left\|\delta_{f} K^{\#}(X)\right\|_{j}^{\prime}\right) G_{j}(X, \phi, 0)
\end{aligned}
$$

and for $\phi=\phi^{\prime}+\zeta$, using ([1], (6.58)) we get:

$$
\begin{aligned}
\left(1+\|\phi\|_{\Phi_{j+1}\left(X^{*}\right)}\right) G_{j}(X, \phi, 0) & \leq\left(1+\|\phi\|_{\Phi_{j+1}\left(X^{*}\right)}\right)^{3} G_{j}(X, \phi, 0) \\
& \leq 4\left(1+\|\phi\|_{\Phi_{j+1}\left(X^{*}\right)}^{3}\right) G_{j}(X, \phi, 0) \\
& \leq 4 q G_{j+1}\left(\bar{X}, \phi^{\prime}, \zeta\right)
\end{aligned}
$$

with $q$ as in ([1, (6.127)). Combining all of them yields

$$
\begin{gathered}
\left\|\left(I-T_{2}\right)_{0} K^{\#}(X, \phi)\right\|_{j+1} \leq 24 q\left(L^{-3 d / 2}\right)\left\|_{0} K^{\#}(X)\right\|_{j}^{\prime} G_{j+1}\left(\bar{X}, \phi^{\prime}, \zeta\right) \\
\left\|\left(I-T_{0}\right) \delta_{f} K^{\#}(X, \phi)\right\|_{j+1} \leq 4 q L^{-d / 2}\left(\left\|\delta_{f} K^{\#}(X)\right\|_{j}^{\prime}\right) G_{j+1}\left(\bar{X}, \phi^{\prime}, \zeta\right)
\end{gathered}
$$

and also using (79) in [5], we obtain:

$$
\begin{aligned}
\left\|\left(I-T_{2}\right)_{0} K^{\#}(X)\right\|_{j+1} & \leq 24 q\left(L^{-3 d / 2}\right)\left\|_{0} K^{\#}(X)\right\|_{j}^{\prime} \\
& \leq 24 q\left(L^{-3 d / 2}\right)(A / 2)^{-|X|_{j}}\left\|_{0} K\right\|_{j} \\
\left\|\left(I-T_{0}\right) \delta_{f} K^{\#}(X)\right\|_{j+1} & \leq 4 q L^{-d / 2}\left\|\delta_{f} K^{\#}(X)\right\|_{j}^{\prime} \\
& \leq 4 q L^{-d / 2}\left(\left\|\delta_{f} K\right\|_{j}\right) A^{-|X|_{j}} 2^{|X|_{j}}
\end{aligned}
$$

Therefore

$$
\begin{aligned}
\left\|\mathcal{L}_{2}\left({ }_{f} K\right)\right\|_{j+1} \leq & \left\|\mathcal{L}_{2}\left({ }_{0} K\right)\right\|_{j+1}+\left\|\mathcal{L}_{2}\left(\delta_{f} K\right)\right\|_{j+1} \\
\leq & 24 q\left(L^{-3 d / 2}\right)\left[\sup _{U} A^{|U|_{j+1}} \sum_{X \in \mathcal{S}_{j}, \bar{X}=U}(A / 2)^{-|X|_{j}}\right]\left\|_{0} K\right\|_{j} \\
& +4 q L^{-d / 2}\left(\left\|\delta_{f} K\right\|_{j}\right) \sup _{U} \sum_{\substack{X \in \mathcal{S}_{j}, \bar{X}=U \\
X^{*} \cap\left\{x_{1}, x_{2}, \ldots x_{m}\right\} \neq \emptyset}} A^{|U|_{j+1}} A^{-|X|_{j}} 2^{|X|_{j}}
\end{aligned}
$$

The bracketed expression is less than $2^{d} 2^{2^{d}} n_{2}(d) L^{d}$ ( using [1], (6.90)) so we have

$$
\left\|\mathcal{L}_{2}\left({ }_{0} K\right)\right\|_{j+1} \leq 24 q 2^{2} 2^{2^{d}} n_{2}(d)\left(L^{-d / 2}\right)\left\|_{0} K\right\|_{j}
$$

Because $|U|_{j+1} \leq|X|_{j} \leq 2^{d}$, we get: 


$$
\begin{gathered}
4 q L^{-d / 2}\left(\left\|\delta_{f} K\right\|_{j}\right) \sup _{U} \sum_{\substack{X \in \mathcal{S}_{j}, \bar{X}=U \\
X^{*} \cap\left\{x_{1}, x_{2}, \ldots x_{m}\right\} \neq \emptyset}} A^{|U|_{j+1}} A^{-|X|_{j}} 2^{|X|_{j}} \\
\leq 4 q L^{-d / 2}\left(\left\|\delta_{f} K\right\|_{j}\right) \sum_{\substack{X \in \mathcal{S}_{j} \\
X^{*} \cap\left\{x_{1}, x_{2}, \ldots x_{m}\right\} \neq \emptyset}} 2^{2^{d}} \\
\leq 4 q L^{-d / 2} 2^{2^{d}}\left(\left\|\delta_{f} K\right\|_{j}\right) \sum_{i=1}^{m} \sum_{\substack{X \in \mathcal{S}_{j}, X^{*} \cap\left\{x_{i}\right\} \neq \emptyset}} 1 \\
\leq 4 q m L^{-d / 2} 2^{2^{d}}\left(\left\|\delta_{f} K\right\|_{j}\right) n_{2}(d)
\end{gathered}
$$

Thus

$$
\left\|\mathcal{L}_{2}\left({ }_{f} K\right)\right\|_{j+1} \leq 24 q 2^{2} 2^{2^{d}} n_{2}(d)\left(L^{-d / 2}\right)\left\|_{0} K\right\|_{j}+L^{-d / 2}\left(\left\|\delta_{f} K\right\|_{j}\right) n_{2}(d) 2^{2^{d}} 4 q m
$$

Moreover $\left\|\delta_{f} K\right\|_{j} \leq\left\|_{0} K\right\|_{j}+\|\|_{f} K \|_{j}$. So we have the Lemma 4

(Q.E.D)

\subsection{Splitting $\mathcal{L}_{3}$}

\subsubsection{Splitting $\mathcal{L}_{3}$}

Similarly in $([5])$, we have a special treatment for the term $\mathcal{L}_{3}$. First we rewrite the final term in $\mathcal{L}_{3}$ which is

$$
\begin{aligned}
& \sum_{\bar{B}=U} \sum_{\substack{X \in \mathcal{S}_{j} \\
X \supset B}} \frac{1}{|X|_{j}}\left[T_{2}\left({ }_{0} K^{\#}(X)\right)+T_{0}\left(\delta_{f} K^{\#}(X)\right)\right] \\
= & \sum_{\bar{B}=U} \sum_{\substack{X \in \mathcal{S}_{j} \\
X \supset B}} \frac{1}{|X|_{j}}\left({ }_{0} K^{\#}(X, 0)+\frac{1}{2}{ }_{0} K_{2}^{\#}(X, 0 ; \phi, \phi)+{ }_{f} K^{\#}(X, 0)-{ }_{0} K^{\#}(X, 0)\right) \\
= & \sum_{\bar{B}=U} \sum_{\substack{X \in \mathcal{S}_{j} \\
X \supset B}} \frac{1}{|X|_{j}}\left({ }_{f} K^{\#}(X, 0)+\frac{1}{2}{ }_{0} K_{2}^{\#}(X, 0 ; \phi, \phi)\right)
\end{aligned}
$$

In ${ }_{0} K_{2}^{\#}(X, 0 ; \phi, \phi)$ we pick a point $z \in B$, then use the same argument as section $4.3[5$ ] by replacing $\phi(x)$ with 6

$$
\phi(z)+\frac{1}{2}(x-z) \cdot \partial \phi(z) \equiv \phi(z)+\frac{1}{2} \sum_{\mu}\left(x_{\mu}-z_{\mu}\right) \partial_{\mu} \phi(z)
$$

\footnotetext{
${ }^{6}$ We need the factor $1 / 2$ since the sum is over $\pm \mu=1, \ldots, d$ and $x_{-\mu}=-x_{\mu}$
} 
If we also average over $z \in B$ (86) becomes

$$
\begin{aligned}
& \sum_{\bar{B}=U} \sum_{\substack{X \in \mathcal{S}_{j} \\
X \supset B}} \frac{1}{|X|_{j}}\left({ }_{f} K^{\#}(X, 0)+\frac{1}{2} \frac{1}{|B|} \sum_{z \in B}{ }_{0} K_{2}^{\#}(X, 0 ; \phi, \phi)\right) \\
= & \sum_{\bar{B}=U} \sum_{\substack{X \in \mathcal{S}_{j} \\
X \supset B}} \frac{1}{|X|_{j}}\left({ }_{f} K^{\#}(X, 0)\right) \\
& +\sum_{\bar{B}=U} \sum_{\substack{X \in \mathcal{S}_{j} \\
X \supset B}} \frac{1}{|X|_{j}}\left(\frac{1}{8|B|} \sum_{z \in B} \sum_{\mu \nu}{ }_{0} K_{2}^{\#}\left(X, 0 ; x_{\mu}, x_{\nu}\right) \partial_{\mu} \phi(z) \partial_{\nu} \phi(z)\right) \\
& +\sum_{\bar{B}=U} \sum_{\substack{X \in \mathcal{S}_{j}: \\
X \supset B}} \frac{1}{|X|_{j}} \sum_{z \in B} \frac{1}{|B|}\left(\frac{1}{2}{ }_{0} K_{2}^{\#}(X, 0 ; \phi, \phi)\right) \\
& -\sum_{\bar{B}=U} \sum_{\substack{X \in \mathcal{S}_{j} \\
X \supset B}} \frac{1}{|X|_{j}}\left(\frac{1}{8|B|} \sum_{z \in B} \sum_{\mu \nu}{ }_{0} K_{2}^{\#}\left(X, 0 ; x_{\mu}, x_{\nu}\right) \partial_{\mu} \phi(z) \partial_{\nu} \phi(z)\right) \\
= & \sum_{\bar{B}=U} \sum_{\substack{X \in \mathcal{S}_{j} \\
X \supset B}} \frac{1}{|X|_{j}}\left({ }_{f} K^{\#}(X, 0)+\frac{1}{8|B|} \sum_{z \in B} \sum_{\mu \nu}{ }_{0} K_{2}^{\#}\left(X, 0 ; x_{\mu}, x_{\nu}\right) \partial_{\mu} \phi(z) \partial_{\nu} \phi(z)\right) \\
& +\mathcal{L}_{3}^{\prime}\left({ }_{f} K\right)(U)
\end{aligned}
$$

here $\mathcal{L}_{3}^{\prime}\left({ }_{f} K\right)(U)=\mathcal{L}_{3}^{\prime}\left({ }_{0} K\right)(U)$ is so called the error, namely

$$
\left.\mathcal{L}_{3}^{\prime}{ }_{0}{ }_{0} K\right)(U)=\sum_{\bar{B}=U} \sum_{\substack{X \in \mathcal{S}_{j}: \\ X \supset B}} \frac{1}{|X|_{j}} \sum_{z \in B} \frac{1}{|B|}\left(\frac{1}{2}{ }_{0} K_{2}^{\#}(X, 0 ; \phi, \phi)-\frac{1}{8}{ }_{0} K_{2}^{\#}(X, 0 ; x \cdot \partial \phi(z), x \cdot \partial \phi(z))\right)
$$

and we can say $\mathcal{L}_{3}^{\prime}\left(\delta_{f} K\right)(U)=0$. Next we define

$$
\begin{gathered}
f \beta(B)={ }_{f} \beta\left({ }_{f} K, B\right)=-\sum_{\substack{X \in \mathcal{S}_{j} \\
X \supset B}} \frac{1}{|X|_{j}}{ }_{f} K^{\#}(X, 0) \\
\alpha_{\mu \nu}(B)=\alpha_{\mu \nu}\left({ }_{f} K, B\right)=\alpha_{\mu \nu}\left({ }_{0} K, B\right)=-\frac{1}{2} \frac{1}{|B|} \sum_{\substack{X \in \mathcal{S}_{j} \\
X \supset B}} \frac{1}{|X|_{j}}{ }_{j} K_{2}^{\#}\left(X, 0 ; x_{\mu}, x_{\nu}\right)
\end{gathered}
$$

Note that $\alpha_{\mu \nu}$ is symmetric and satisfies $\alpha_{-\mu \nu}=-\alpha_{\mu \nu}$. We also let $\alpha_{\mu \nu}$ stand for the function $\alpha_{\mu \nu}(x)$ which takes the constant value $\alpha_{\mu \nu}(B)$ for $x \in B$.

Now we write (87) as

$$
\begin{aligned}
& \sum_{B=U} \sum_{\substack{X \in \mathcal{S}_{j} \\
X \supset B}} \frac{1}{|X|_{j}}\left({ }_{f} K^{\#}(X, 0)+\frac{1}{8|B|} \sum_{z \in B} \sum_{\mu \nu}{ }_{0} K_{2}^{\#}\left(X, 0 ; x_{\mu}, x_{\nu}\right) \partial_{\mu} \phi(z) \partial_{\nu} \phi(z)\right)+\mathcal{L}_{3}^{\prime}\left({ }_{0} K\right)(U) \\
= & \sum_{\bar{B}=U}\left(\frac{1}{4} \sum_{z \in B} \sum_{\mu \nu} \frac{1}{2} \frac{1}{|B|} \sum_{\substack{X \in \mathcal{S}_{j} \\
X \supset B}} \frac{1}{|X|_{j}}{ }_{0} K_{2}^{\#}\left(X, 0 ; x_{\mu}, x_{\nu}\right)(B) \partial_{\mu} \phi(z) \partial_{\nu} \phi(z)\right) \\
& +\sum_{\bar{B}=U} \sum_{\substack{X \in \mathcal{S}_{j} \\
X \supset B}} \frac{1}{|X|_{j}} f K^{\#}(X, 0)+\mathcal{L}_{3}^{\prime}\left({ }_{0} K\right)(U)
\end{aligned}
$$




$$
\begin{aligned}
& \left.=-\sum_{\bar{B}=U}\left({ }_{f} \beta(B)+\frac{1}{4} \sum_{z \in B} \sum_{\mu \nu} \alpha_{\mu \nu}(B) \partial_{\mu} \phi(z) \partial_{\nu} \phi(z)\right)+\mathcal{L}_{3}^{\prime}{ }_{0} K\right)(U) \\
& \left.=-\left(\sum_{\bar{B}=U} V\left({ }_{f} \beta, \alpha, B, \phi\right)\right)+\mathcal{L}_{3}^{\prime}{ }_{0} K\right)(U)
\end{aligned}
$$

where $V\left({ }_{f} \beta, \alpha, B, \phi\right)$ defined in (35) $)$. Combining all of the above, we get:

$$
\mathcal{L}_{3}\left({ }_{f} E, \sigma,{ }_{f} E, \tilde{\sigma},{ }_{f} K,{ }_{0} K\right)(U)=\sum_{\bar{B}=U}\left(V\left({ }_{f} E, \tilde{\sigma}, B\right)-V^{\#}\left({ }_{f} E, \sigma, B\right)-V\left({ }_{f} \beta, \alpha, B\right)\right)+\mathcal{L}_{3}^{\prime}\left({ }_{0} K\right)(U)
$$

\subsubsection{Estimating $\alpha,{ }_{f} \beta$ and $\mathcal{L}_{3}^{\prime}$}

First we find some explicit upper bounds for $\alpha$ and ${ }_{f} \beta$

Lemma 5 (Estimates $\beta$ and $\alpha$ )

$$
\begin{gathered}
\left\|_{f} \beta\right\|_{j} \equiv \sup _{B \in \mathcal{B}_{j}}\left|{ }_{f} \beta(B)\right| \leq 2 n_{2}(d) A^{-1}\left\|_{f} K\right\|_{j} \\
\|\alpha\|_{j} \equiv \sup _{B \in \mathcal{B}_{j}} \sum_{\mu \nu}\left|\alpha_{\mu \nu}(B)\right| \leq 4(2 d)^{2} n_{2}(d) h^{-2} A^{-1}\left\|_{0} K\right\|_{j}
\end{gathered}
$$

Remark. The norm $\|\alpha\|_{j}$ agrees with the norm $\|s\|_{j}$ in (39) if $s_{\mu \nu}(x)=\alpha_{\mu \nu}(B)$ for $x \in B$.

Proof. By (70) and (79) in [5], with $A$ very large, we have:

$$
\begin{aligned}
\left|{ }_{f} K^{\#}(X, 0)\right| & \leq\left\|_{f} K^{\#}(X)\right\|_{j}^{\prime} \leq(A / 2)^{-1}\left\|_{f} K\right\|_{j} \\
\left\|_{0} K_{2}^{\#}(X, 0)\right\|_{j} & \leq 2\left\|_{0} K^{\#}(X)\right\|_{j}^{\prime} \leq 4 A^{-1}\left\|_{0} K\right\|_{j}
\end{aligned}
$$

From (30), the number of small sets containing a block $B$ is $n_{2}(d)$ which is bounded and depends only on $d$, we have:

$$
\begin{aligned}
\left|{ }_{f} \beta(B)\right| & \leq \sum_{X \in \mathcal{S}_{j}, X \supset B}\left|{ }_{f} K^{\#}(X, 0)\right| \\
& \leq \sum_{X \in \mathcal{S}_{j}, X \supset B} 2 A^{-1}\left\|_{f} K\right\|_{j} \\
& \leq 2 n_{2}(d) A^{-1}\left\|_{f} K\right\|_{j}
\end{aligned}
$$

We also have $\left\|x_{\mu}\right\|_{\Phi_{j}\left(X^{*}\right)}=h^{-1} L^{d j / 2}$ and $|B|=L^{d j}$. By using (66) in [5] we get

$$
\begin{aligned}
\left.|B|^{-1}\right|_{0} K_{2}^{\#}\left(X, 0 ; x_{\mu}, x_{\nu}\right) \mid & \leq\left(h^{-1} L^{d j / 2}\right)^{2} L^{-d j}\left\|_{0} K_{2}^{\#}(X, 0)\right\|_{j} \\
& =h^{-2}\left\|_{0} K_{2}^{\#}(X, 0)\right\|_{j} \\
& \leq 4 h^{-2} A^{-1}\left\|_{0} K\right\|_{j}
\end{aligned}
$$

then

$$
\begin{aligned}
\sum_{\mu \nu}\left|\alpha_{\mu \nu}(B)\right| & \leq\left.\sum_{\mu \nu} \sum_{X \in \mathcal{S}_{j}, X \supset B}|B|^{-1}\right|_{0} K_{2}^{\#}\left(X, 0 ; x_{\mu}, x_{\nu}\right) \mid \\
& \leq \sum_{\mu \nu} \sum_{X \in \mathcal{S}_{j}, X \supset B} 4 h^{-2} A^{-1}\left\|_{0} K\right\|_{j} \\
& \leq \sum_{\mu \nu} n_{2}(d) 4 h^{-2} A^{-1}\left\|_{0} K\right\|_{j} \\
& \leq(2 d)^{2} n_{2}(d) 4 h^{-2} A^{-1}\left\|_{0} K\right\|_{j}
\end{aligned}
$$


Now we give some estimate for $\mathcal{L}_{3}^{\prime}$

Lemma 6 Let $L$ be sufficiently large. Then the operator $\mathcal{L}_{3}^{\prime}$ is a contraction with arbitrarily small norm.

$$
\left\|\mathcal{L}_{3}^{\prime}\left({ }_{0} K\right)\right\|_{j+1} \leq 72 d^{2} 2^{2 d} n_{1}(d)\left(L^{-2}\right)\left\|_{0} K\right\|_{j}
$$

Proof. Based on the proof of Lemma 8 in [5], we make some modifications and obtain a better upper bound with some explicit coefficient.

We have

$$
\left.\mathcal{L}_{3}^{\prime}{ }_{0} K\right)(U)=\sum_{\bar{B}=U} \sum_{\substack{X \in \mathcal{S}_{j} \\ X \supset B}} \frac{1}{|X|_{j}} \sum_{z \in B} \frac{1}{|B|} \frac{1}{2}{ }_{0} K_{2}^{\#}\left(X, 0 ; \phi-\frac{1}{2} x \cdot \partial \phi(z), \phi+\frac{1}{2} x \cdot \partial \phi(z)\right)
$$

Using ([5], (152)- (154)) we get:

$$
\begin{aligned}
& \left\|\phi-\frac{1}{2} x \cdot \partial \phi(z)\right\|_{\Phi_{j}\left(X^{*}\right)} \leq 3 d 2^{d}\left(L^{-d / 2-1}\right)\|\phi\|_{\Phi_{j+1}\left(X^{*}\right)} \\
& \left\|\phi+\frac{1}{2} x \cdot \partial \phi(z)\right\|_{\Phi_{j}\left(X^{*}\right)} \leq 3 d 2^{d}\left(L^{-d / 2-1}\right)\|\phi\|_{\Phi_{j+1}\left(X^{*}\right)}
\end{aligned}
$$

Now we estimate

$$
{ }_{0} H_{X}(U, \phi)={ }_{0} K_{2}^{\#}\left(X, 0 ; \phi-\frac{1}{2} x \cdot \partial \phi(z), \phi+\frac{1}{2} x \cdot \partial \phi(z)\right)
$$

Using the same argument as (156)-(157) in [5], we obtain:

$$
\left\|_{0} H_{X}(U, \phi)\right\|_{j+1} \leq 18 d^{2} 2^{2 d}\left(L^{-d-2}\right)\left\|K_{2}^{\#}(X, 0)\right\|_{j}\left(1+\|\phi\|_{\Phi_{j+1}\left(U^{*}\right)}^{2}\right)
$$

But for $\phi=\phi^{\prime}+\zeta$

$$
\left(1+\|\phi\|_{\Phi_{j+1}\left(U^{*}\right)}^{2}\right) \leq G_{s, j+1}(U, \phi, 0) \leq G_{s, j+1}^{2}\left(U, \phi^{\prime}, \zeta\right) \leq G_{j+1}\left(U, \phi^{\prime}, \zeta\right)
$$

Also using (94) we can get:

$$
\left\|H_{X}(U)\right\|_{j+1} \leq 72 d^{2} 2^{2 d}\left(L^{-d-2}\right) A^{-1}\|K\|_{j}
$$

which yields to

$$
\begin{aligned}
\left\|\mathcal{L}_{3}^{\prime} K(U)\right\|_{j+1} & \leq n_{1}(d) \sum_{\bar{B}=U}\left\|H_{X}(U)\right\|_{j+1} \\
& \leq n_{1}(d) L^{d} 72 d^{2} 2^{2 d}\left(L^{-d-2}\right) A^{-1}\|K\|_{j} \\
& \leq 72 d^{2} 2^{2 d} n_{1}(d)\left(L^{-2}\right) A^{-1}\|K\|_{j}
\end{aligned}
$$

Since $\mathcal{L}_{3}^{\prime} K(U)$ is zero unless $|U|_{j+1}=1$ this gives

$$
\left\|\mathcal{L}_{3}^{\prime} K\right\|_{j+1} \leq 72 d^{2} 2^{2 d} n_{1}(d)\left(L^{-2}\right)\|K\|_{j}
$$

(Q.E.D) 


\subsection{Identifying invariant parts and estimating the others}

N0w we investigate the 1 st term of (92). We notice that $\alpha_{\mu \nu}(B)=\alpha_{\mu \nu}\left({ }_{f} K, B\right)=\alpha_{\mu \nu}\left({ }_{0} K, B\right)$ is independent from $f(\phi)$ and ${ }_{0} E(B),{ }_{0} K(X, \phi)$ actually is the same as $E(B), K(X, \phi)$ in lemma $9[5]$. Therefore we have the same result as lemma 9 [5]

Lemma 7 (Lemma 9, Dimock [5])

Suppose ${ }_{0} E(B),{ }_{0} K(X, \phi)$ are invariant under lattice symmetries away from the boundary of $\Lambda_{N}$ and ${ }_{0} \tilde{E}(B)$ is invariant for $B^{*}$ away from the boundary. Then

1. ${ }_{0} E^{\prime}\left(B^{\prime}\right),{ }_{0} K^{\prime}(U, \phi)$ are invariant for $B^{\prime}, U$ away from the boundary

2. If $B^{*}$ is away from the boundary then ${ }_{0} \beta(B), \alpha_{\mu \nu}(B)$ are independent of $B$ and $\alpha_{\mu \nu}(B)=\hat{\alpha}_{\mu \nu}(B)$ defined for all $B$ by

$$
\hat{\alpha}_{\mu \nu}(B)=\frac{\alpha}{2}\left(\delta_{\mu \nu}-\delta_{\mu,-\nu}\right)
$$

where $\alpha$ is a constant.

For all $B \in \mathcal{B}_{j}$ we define

$$
\alpha_{\mu \nu}^{\prime}(B)=\alpha \delta_{\mu \nu}
$$

and write, for any $U \in \mathcal{B}_{j+1}$

$$
\sum_{\bar{B}=U} V\left({ }_{f} \beta, \alpha, B\right)=\sum_{\bar{B}=U} V\left({ }_{f} \beta, \alpha^{\prime}, B\right)-\mathcal{L}_{4}\left({ }_{f} K\right)(U)-\Delta\left({ }_{f} K\right)(U)
$$

where, for $U \subset \mathcal{B}_{j+1}$,

$$
\begin{aligned}
\mathcal{L}_{4}\left({ }_{f} K\right)(U) & =\mathcal{L}_{4}\left({ }_{0} K\right)(U)=\sum_{\bar{B}=U} V\left(0, \alpha^{\prime}-\hat{\alpha}, B\right)=V\left(0, \alpha^{\prime}-\hat{\alpha}, U\right) \\
\Delta\left({ }_{f} K\right)(U) & =\Delta\left({ }_{0} K\right)(U)=\sum_{\bar{B}=U} V(0, \hat{\alpha}-\alpha, B)=V(0, \tilde{\alpha}, U)
\end{aligned}
$$

where $\tilde{\alpha}_{\mu \nu}(x)=\hat{\alpha}_{\mu \nu}(B)-\alpha_{\mu \nu}(B)$ if $x \in B$. Then we can write that $\mathcal{L}_{4}\left(\delta_{f} K\right)(U)=0$ and $\Delta\left(\delta_{f} K\right)(U)=$ 0

By the above definition $\Delta\left({ }_{0} K\right)(U)$ vanishes unless $U$ touches the boundary. Now (92) becomes

$$
\begin{aligned}
& \mathcal{L}_{3}\left({ }_{f} E, \sigma,{ }_{f} E, \tilde{\sigma},{ }_{f} K,{ }_{0} K\right)(U) \\
& =\sum_{\bar{B}=U}\left(V\left({ }_{f} \tilde{E}, \tilde{\sigma}, B\right)-V^{\#}\left({ }_{f} E, \sigma, B\right)-V\left({ }_{f} \beta, \alpha^{\prime}, B\right)\right) \\
& \quad+\mathcal{L}_{3}^{\prime}\left({ }_{0} K\right)(U)+\mathcal{L}_{4}\left({ }_{0} K\right)(U)+\Delta\left({ }_{0} K\right)(U)
\end{aligned}
$$

Remark. Because $\mathcal{L}_{4}\left({ }_{f} K\right)=\mathcal{L}_{4}\left({ }_{0} K\right)$ and $\Delta\left({ }_{f} K\right)=\Delta\left({ }_{0} K\right)$ are independent from $f$, we will have the same results as Lemma 10 and Lemma 11 in (Dimock, [5). Moreover, by using Lemma 5 above, we can obtain some explicit upper bounds for $\mathcal{L}_{4}\left({ }_{0} K\right)$ and $\Delta\left({ }_{0} K\right)$.

Lemma 8 Let $L$ be sufficiently large. Then the operator $\mathcal{L}_{4}$ is a contraction with

$$
\left\|\mathcal{L}_{4}\left({ }_{0} K\right)\right\|_{j+1} \leq 4(2 d)^{3} n_{2}(d) L^{-(j+1)}\left\|_{0} K\right\|_{j}
$$

Lemma 9 Let $L$ be sufficiently large. Then the operator $\Delta$ is a contraction with

$$
\left\|\Delta\left({ }_{0} K\right)\right\| \leq 4(2 d)^{5} 2^{d} n_{2}(d) L^{-1}\left\|_{0} K\right\|_{j}
$$




\subsection{Simplifying for the next scale}

We now pick ${ }_{f} \tilde{E}(B), \tilde{\sigma}$ so the $V$ terms in (111) cancel. We have:

$$
\begin{aligned}
V^{\#}\left({ }_{f} E, \sigma, B, \phi\right)= & { }_{f} E(B)+\int \frac{\sigma}{4} \sum_{x \in B} \sum_{\mu}\left(\partial_{\mu} \phi(x)+\partial_{\mu} \zeta(x)\right)^{2} d \mu_{\Gamma_{j+1}}(\zeta) \\
= & { }_{f} E(B)+\frac{\sigma}{4} \sum_{x \in B} \sum_{\mu} \partial_{\mu} \phi(x)^{2} \int d \mu_{\Gamma_{j+1}}(\zeta) \\
& +\frac{\sigma}{2} \sum_{x \in B} \sum_{\mu} \partial_{\mu} \phi(x) \int \partial_{\mu} \zeta(x) d \mu_{\Gamma_{j+1}}(\zeta) \\
& +\frac{\sigma}{4} \sum_{x \in B} \sum_{\mu} \int \partial_{\mu} \zeta(x)^{2} d \mu_{\Gamma_{j+1}}(\zeta) \\
= & f E(B)+\frac{\sigma}{4} \sum_{x \in B} \sum_{\mu} \partial_{\mu} \phi(x)^{2}+0+\frac{\sigma}{4} \sum_{x \in B} \sum_{\mu}\left(\partial_{\mu} \Gamma_{j+1} \partial_{\mu}^{*}\right)(x, x) \\
\equiv & V\left({ }_{f} E, \sigma, B, \phi\right)+\frac{\sigma}{4} \sum_{\mu} \operatorname{Tr}\left(1_{B} \partial_{\mu} \Gamma_{j+1} \partial_{\mu}^{*}\right)
\end{aligned}
$$

because

$$
\begin{aligned}
\int \partial_{\mu} \zeta(x) d \mu_{\Gamma_{j+1}}(\zeta) & =0 \\
\int \partial_{\mu} \zeta(x)^{2} d \mu_{\Gamma_{j+1}}(\zeta) & =\int\left(\zeta, \partial_{\mu}^{*} \delta_{x}\right)\left(\zeta, \partial_{\mu}^{*} \delta_{x}\right) d \mu_{\Gamma_{j+1}}(\zeta) \\
& =\left(\partial_{\mu}^{*} \delta_{x}, \Gamma_{j+1} \partial_{\mu}^{*} \delta_{x}\right) \\
& =\left(\delta_{x}, \partial_{\mu} \Gamma_{j+1} \partial_{\mu}^{*} \delta_{x}\right) \\
& =\left(\partial_{\mu} \Gamma_{j+1} \partial_{\mu}^{*}\right)(x, x)
\end{aligned}
$$

If we choose ${ }_{f} \tilde{E}={ }_{f} \tilde{E}\left({ }_{f} E, \sigma,{ }_{f} K\right)$

$$
\tilde{f} E(B)={ }_{f} E(B)+\frac{\sigma}{4} \sum_{\mu} \operatorname{Tr}\left(1_{B} \partial_{\mu} \Gamma_{j+1} \partial_{\mu}^{*}\right)+{ }_{f} \beta\left({ }_{f} K, B\right)
$$

then the constant terms of (114) will be canceled. The second order terms of (114) would be vanish if we define $\tilde{\sigma}=\tilde{\sigma}\left(\sigma,{ }_{f} K\right)=\tilde{\sigma}\left(\sigma,{ }_{0} K\right)$ by

$$
\tilde{\sigma}=\sigma+\alpha\left({ }_{f} K\right)=\sigma+\alpha\left({ }_{0} K\right)
$$

Here we are canceling the constant term exactly for all $B$, but for the quadratic term we only cancel the invariant version away from the boundary.

By composing ${ }_{f} K^{\prime}={ }_{f} K^{\prime}\left({ }_{f} \tilde{E}, \tilde{\sigma},{ }_{f} E, \sigma,{ }_{f} K,{ }_{0} K\right)$ in theorem5 5 with newly defined ${ }_{f} \tilde{E}={ }_{f} \tilde{E}\left({ }_{f} E, \sigma,{ }_{f} K\right)$ and $\tilde{\sigma}=\tilde{\sigma}\left(\sigma,{ }_{f} K\right)=\tilde{\sigma}\left(\sigma,{ }_{0} K\right)$ we obtain a new map ${ }_{f} K^{\prime}={ }_{f} K^{\prime}\left({ }_{f} E, \sigma,{ }_{f} K,{ }_{0} K\right)$. We also have new quantities ${ }_{f} E^{\prime}\left({ }_{f} E, \sigma,{ }_{f} K\right)$ defined by ${ }_{f} E^{\prime}\left(B^{\prime}\right)=\sum_{B \subset B^{\prime} f} \tilde{E}(B)$ and $\sigma^{\prime}=\sigma^{\prime}\left(\sigma,{ }_{f} K\right)=\sigma^{\prime}\left(\sigma,{ }_{0} K\right)$ defined by $\sigma^{\prime}=\tilde{\sigma}=\sigma+\alpha\left({ }_{f} K\right)=\sigma+\alpha\left({ }_{0} K\right)$ as normal. These quantities satisfy (45)

$$
\mu_{\Gamma_{j+1}} *\left({ }_{f} I\left({ }_{f} E, \sigma\right) \circ{ }_{f} K\right)(\Lambda)=\left({ }_{f} I^{\prime}\left({ }_{f} E^{\prime}, \sigma^{\prime}\right) \circ{ }_{f} K^{\prime}\right)(\Lambda)
$$

Here we still assume that $L$ is sufficiently large, and that $A$ is sufficiently large depending on $L$.

Theorem 6 1. For $R>0$ there is a $r>0$ such that the following holds for all $j$. If $\left\|_{f} E\right\|_{j}$, $|\sigma|, \max \left\{\left\|_{f} K\right\|_{j},\left\|_{0} K\right\|_{j}\right\}<r$ then $\left\|_{f} E^{\prime}\right\|_{j+1},\left|\sigma^{\prime}\right|, \max \left\{\|\|_{f} K^{\prime}\left\|_{j+1},\right\|_{0} K^{\prime} \|_{j+1}\right\}<R$. Furthermore ${ }_{f} E^{\prime},{ }_{f} K^{\prime}, \sigma^{\prime}$ are smooth functions of ${ }_{f} E, \sigma,{ }_{f} K,{ }_{0} K$ on this domain with derivatives bounded uniformly in $j$. The analyticity of ${ }_{f} K^{\prime}$ in $t_{1}, \ldots, t_{m}$ still holds when we go from $j$-scale to $(j+1)$ scale. 
2. The linearization of ${ }_{f} K^{\prime}={ }_{f} K^{\prime}\left({ }_{f} E, \sigma,{ }_{f} K,{ }_{0} K\right)$ at the origin is the contraction $\mathcal{L}\left({ }_{f} K\right)$ where

$$
\mathcal{L}=\mathcal{L}_{1}+\mathcal{L}_{2}+\mathcal{L}_{3}^{\prime}+\mathcal{L}_{4}+\Delta
$$

Proof. For the first part, by combining with theorem 5, it suffices to show that the linear maps ${ }_{f} \tilde{E}$ and $\tilde{\sigma}$ have norms bounded uniformly in $j$. Using the estimate $\left|\alpha\left({ }_{f} K\right)\right|=\left|\alpha\left({ }_{0} K\right)\right| \leq$ $4(2 d)^{2} n_{2}(d) h^{-2} A^{-1}\left\|_{0} K\right\|_{j}$ from lemma 5 , we have $\tilde{\sigma}$ is bounded. From lemma 5 we also have the bound on $\left\|_{f} \beta\left({ }_{f} K\right)\right\|_{j} \leq 2 n_{2}(d) A^{-1}\left\|_{f} K\right\|_{j}$. For $B \in \mathcal{B}_{j}$, the estimate (19) gives us

$$
\mid \frac{\sigma}{4} \sum_{\mu} \operatorname{Tr}\left(1_{B}\left(\partial_{\mu} \Gamma_{j+1} \partial_{\mu}^{*}\right)\left|\leq d c_{1,1}\right| \sigma\left|\sum_{x \in B} L^{-d j} \leq d c_{1,1}\right| \sigma \mid\right.
$$

where $c_{1,1}$ as in (19). Combining with (116) we have that ${ }_{f} \tilde{E}={ }_{f} E\left({ }_{f} E, \sigma,{ }_{f} K\right)$ satisfies

$$
\left\|_{f} \tilde{E}\right\|_{j} \leq\left\|_{f} E\right\|_{j}+\mathcal{C}\left(|\sigma|+A^{-1}\left\|_{f} K\right\|_{j}\right)
$$

where $\mathcal{C}=\max \left\{d c_{1,1}, 2 n_{2}(d)\right.$.

The second part follows since the linearization of the new function ${ }_{f} K^{\prime}$ is the linearization of the old function ${ }_{f} K^{\prime}$ in theorem 5 composed with ${ }_{f} E={ }_{f} E\left({ }_{f} E, \sigma,{ }_{f} K\right), \tilde{\sigma}=\tilde{\sigma}\left(\sigma,{ }_{f} K\right)=\tilde{\sigma}\left(\sigma,{ }_{0} K\right)$. (All of them vanish at zero.) The cancellation gives us only with $\mathcal{L}\left({ }_{f} K\right)$.

\subsection{Forming RG FLow}

It is easier for us if we can extract the energy from the other variables. Assume that we start with $E(B)=0$ in (118)

$$
\mu_{\Gamma_{j+1}} *\left({ }_{f} I(0, \sigma) \circ{ }_{f} K\right)\left(\Lambda_{N}\right)=\left({ }_{f} I^{\prime}\left({ }_{f} E^{\prime}, \sigma^{\prime}\right) \circ{ }_{f} K^{\prime}\right)(\Lambda)
$$

where $\sigma^{\prime}=\sigma^{\prime}\left(\sigma,{ }_{f} K\right)=\sigma^{\prime}\left(\sigma,{ }_{0} K\right)$ and ${ }_{f} K^{\prime}={ }_{f} K^{\prime}\left(0, \sigma,{ }_{f} K\right)$ and ${ }_{f} E^{\prime}={ }_{f} E^{\prime}\left(0, \sigma,{ }_{f} K\right)$ as above. Then we remove the ${ }_{f} E^{\prime}$ by making an adjustment in ${ }_{f} K^{\prime}$.

$$
\begin{aligned}
& \mu_{\Gamma_{j+1}} *\left({ }_{f} I(0, \sigma) \circ{ }_{f} K\right)\left(\Lambda_{N}\right)=\left({ }_{f} I^{\prime}\left({ }_{f} E^{\prime}, \sigma^{\prime}\right) \circ{ }_{f} K^{\prime}\right)(\Lambda) \\
& =\sum_{U \in \mathcal{P}_{j+1}}\left({ }_{f} I^{\prime}\left({ }_{f} E^{\prime}, \sigma^{\prime}\right)(\Lambda-U)\right)\left({ }_{f} K^{\prime}\left(0, \sigma,{ }_{f} K, U\right)\right) \\
& =\sum_{U \in \mathcal{P}_{j+1}}\left(\prod_{B^{\prime} \in \mathcal{B}_{j+1}(\Lambda-U)}{ }_{f} I^{\prime}\left({ }_{f} E^{\prime}, \sigma^{\prime}\right)\left(B^{\prime}\right)\right)\left({ }_{f} K^{\prime}\left(0, \sigma,{ }_{f} K, U\right)\right) \\
& =\sum_{U \in \mathcal{P}_{j+1}}\left(\prod_{B^{\prime} \in \mathcal{B}_{j+1}(\Lambda-U)} \exp \left({ }_{f} E^{\prime}\left(B^{\prime}\right)\right)\left[{ }_{f} I^{\prime}\left(0, \sigma^{\prime}\right)\left(B^{\prime}\right)\right]\right)\left({ }_{f} K^{\prime}\left(0, \sigma,{ }_{f} K, U\right)\right) \\
& \left.=\sum_{U \in \mathcal{P}_{j+1}}\left(\sum_{B^{\prime} \in \mathcal{B}_{j+1}(\Lambda-U)}{ }_{f} E^{\prime}\left(B^{\prime}\right)\right){ }_{f} I^{\prime}\left(0, \sigma^{\prime}\right)(\Lambda-U)\right)\left({ }_{f} K^{\prime}\left(0, \sigma,{ }_{f} K, U\right)\right) \\
& =\exp \left(\sum_{B^{\prime} \in \mathcal{B}_{j+1}\left(\Lambda_{N}\right)}\left({ }_{f} E^{+}\left(B^{\prime}\right)\right)\left({ }_{f} I^{\prime}\left(0, \sigma^{+}\right) \circ{ }_{f} K^{+}\right)\left(\Lambda_{N}\right)\right.
\end{aligned}
$$

where ${ }_{f} E^{+}\left(\sigma,{ }_{f} K, B^{\prime}\right), \sigma^{+}\left(\sigma,{ }_{f} K\right),{ }_{f} K^{+}\left(\sigma,{ }_{f} K, U\right)$ are defined as following $\left(U \in \mathcal{P}_{j+1}, B^{\prime} \in \mathcal{B}_{j+1}\right)$

$$
\begin{aligned}
{ }_{f} E^{+}\left(\sigma,{ }_{f} K, B^{\prime}\right) & \equiv{ }_{f} E^{\prime}\left(0, \sigma,{ }_{f} K, B^{\prime}\right)=\sum_{B \subset B^{\prime}} \tilde{f} E\left(0, \sigma,{ }_{f} K, B\right) \\
\sigma^{+}\left(\sigma,{ }_{f} K\right) & \equiv \sigma^{\prime}\left(\sigma,{ }_{f} K\right)=\sigma^{\prime}\left(\sigma,{ }_{0} K\right)=\sigma+\alpha\left({ }_{0} K\right) \\
{ }_{f} K^{+}\left(\sigma,{ }_{f} K, U\right) & \equiv \exp \left(-\sum_{B^{\prime} \in \mathcal{B}_{j+1}(U)}{ }_{f} E^{+}\left(B^{\prime}\right)\right){ }_{f} K^{\prime}\left(0, \sigma,{ }_{f} K, U\right)
\end{aligned}
$$


The dynamical variables are now $\sigma^{+}\left(\sigma,{ }_{f} K\right)$ and ${ }_{f} K^{+}\left(\sigma,{ }_{f} K\right)$. The extracted energy ${ }_{f} E^{+}(\sigma, K)$ is controlled by the other variables. Because everything vanishes at the origin the linearization of ${ }_{f} K^{+}\left(\sigma,{ }_{f} K\right)$ is still $\mathcal{L}\left({ }_{f} K\right)$. The bound (121) on ${ }_{f} E$ would give us an upper bound on ${ }_{f} E^{+}$and our theorem 6 becomes:

Theorem 7 1. For $R>0$ there is a $r>0$ such that the following holds for all $j$. If $|\sigma|$, $\max \left\{\left\|_{f} K\right\|_{j},\left\|_{0} K\right\|_{j}\right\}<r$ then $\left|\sigma^{+}\right|, \max \left\{\|\|_{f} K^{+}\left\|_{j+1},\right\|_{0} K^{+} \|_{j+1}\right\}<R$. Furthermore $\sigma^{+},{ }_{f} K^{+}$ are smooth functions of $\sigma,{ }_{f} K$ on this domain with derivatives bounded uniformly in $j$. The analyticity of ${ }_{f} K^{+}$in $t_{1}, \ldots, t_{m}$ still holds when we go from $j$-scale to $(j+1)$-scale.

2. The extracted energies satisfy

$$
\left\|_{f} E^{+}\left(\sigma,{ }_{f} K\right)\right\|_{j+1} \leq \mathcal{C}\left(L^{d}\right)\left(|\sigma|+A^{-1}\left\|_{f} K\right\|_{j}\right)
$$

3. The linearization of $K^{+}$at the origin is the contraction $\mathcal{L}$.

\section{The stable manifold}

Up to now, we have not specialized to the dipole gas, but take a general initial point $\sigma_{0},{ }_{f} K_{0}$ corresponding to an integral $\int\left({ }_{f} I\left(0, \sigma_{0}\right){ }_{f} K_{0}\right)\left(\Lambda_{N}\right) d \mu_{C_{0}}$. We assume ${ }_{0} K_{0}(X, \phi)$ has the lattice symmetries and satisfies the conditions (37). We also assume $\left|\sigma_{0}\right|, \max \left\{\left\|_{f} K\right\|_{0},\left\|_{0} K\right\|_{0}\right\}<r$ where $r$ is small enough so the theorem 7 holds, say with $R=1$, then we can take the first step. We apply the transformation (123) for $j=0,1,2, \ldots$ and continue as far as we can. Then we get a sequence $\sigma_{j},{ }_{f} K_{j}^{N}(X)$ by $\sigma_{j+1}=\sigma^{+}\left(\sigma_{j},{ }_{f} K_{j}^{N}\right)$ and ${ }_{f} K_{j+1}^{N}={ }_{f} K^{+}\left(\sigma_{j},{ }_{f} K_{j}^{N}\right)$ with extracted energies ${ }_{f} E_{j+1}^{N}={ }_{f} E^{+}\left(\sigma_{j},{ }_{f} K_{j}^{N}\right)$. Then we have, for any $l$, with ${ }_{f} I_{j}\left(\sigma_{j}\right)={ }_{f} I_{j}\left(0, \sigma_{j}\right)$

$$
\int\left({ }_{f} I_{0}\left(\sigma_{0}\right) \circ{ }_{f} K_{0}\right)\left(\Lambda_{N}\right) d \mu_{C_{0}}=\exp \left(\sum_{j=1}^{l} \sum_{B \in \mathcal{B}_{j}\left(\Lambda_{N}\right)}{ }_{f} E_{j}^{N}(B)\right) \int\left({ }_{f} I_{l}\left(\sigma_{l}\right) \circ{ }_{f} K_{l}^{N}\right)\left(\Lambda_{N}\right) d \mu_{C_{l}}
$$

The quantities ${ }_{0} K_{j}^{N}(X)$ and ${ }_{0} E_{j}^{N}(B)$ are independent of $N$ and have the lattice symmetries if $X, B$ are away from the boundary $\partial \Lambda_{N}$ in the sense that they have no boundary blocks. These properties are true initially and are preserved by the iteration. In these cases we denote these quantities by just ${ }_{0} K_{j}(X)$ and ${ }_{0} E_{j}(B)$

With our construction $\alpha$ defined in (89), (107) only depends on ${ }_{0} K_{j}$. By splitting $K^{+}$into a linear and a higher order piece the sequence $\sigma_{j},{ }_{f} K_{j}^{N}(X)$ is generated by the RG transformation

$$
\begin{aligned}
\sigma_{j+1} & =\sigma_{j}+\alpha\left(K_{j}\right) \\
{ }_{0} K_{j+1}^{N} & =\mathcal{L}\left({ }_{0} K_{j}^{N}\right)+{ }_{0} g\left(\sigma_{j},{ }_{0} K_{j}^{N}\right) \\
\delta_{f} K_{j+1}^{N} & =\left(\mathcal{L}_{1}+\mathcal{L}_{2}\right)\left(\delta_{f} K_{j}^{N}\right)+{ }_{f} g\left(\sigma_{j},{ }_{f} K_{j}^{N},{ }_{0} K_{j}^{N}\right)-{ }_{0} g\left(\sigma_{j},{ }_{0} K_{j}^{N}\right)
\end{aligned}
$$

This is regarded as a mapping from the Banach space $\mathbb{R} \times\left(\mathcal{K}_{j}\left(\Lambda_{N}\right) \times \mathcal{K}_{j}\left(\Lambda_{N}\right)\right)$ to the Banach space $\mathbb{R} \times\left(\mathcal{K}_{j}\left(\Lambda_{N}\right) \times \mathcal{K}_{j}\left(\Lambda_{N}\right)\right)$. The 2 nd equation of (127) defines ${ }_{0} g$ which is smooth with derivatives bounded uniformly in $j$ and satisfies ${ }_{0} g(0,0)=0, D\left({ }_{0} g\right)(0,0)=0$. The last equation of (127) defines ${ }_{f} g$ which is also smooth with derivatives bounded uniformly in $j$ and satisfies ${ }_{f} g(0,0)=0, D\left({ }_{f} g\right)(0,0)=0$.

Now we consider the first two equations in (127). Around the origin there are a neutral direction $\sigma_{j}$ and a contracting direction $K_{j}$ (since $\mathcal{L}$ is a contraction.). Hence we expect there is a stable manifold. We quote a version of the stable manifold theorem due to Brydges [1, as applied in Theorem 7 in Dimock [5] 
Theorem 8 (Theorem 7, Dimock [5])

Let $L$ be sufficiently large, A sufficiently large (depending on $L$ ), and $r$ sufficiently small (depending on $L, A)$. Then there is $0<\tau<r$ and a smooth real-valued function $\sigma_{0}=h\left({ }_{0} K_{0}\right), h(0)=0$, mapping $\left\|_{0} K_{0}\right\|_{0}<\tau$ into $\left|\sigma_{0}\right|<r$ such that with these start values the sequence $\sigma_{j},{ }_{0} K_{j}^{N}$ is defined for all $0 \leq j \leq N$ and

$$
\left|\sigma_{j}\right| \leq r 2^{-j} \quad\left\|_{0} K_{j}^{N}\right\|_{j} \leq r 2^{-j}
$$

Furthermore the extracted energies satisfy

$$
\left\|_{0} E_{j+1}^{N}\right\|_{j+1} \leq 2 \mathcal{C}\left(L^{d}\right) r 2^{-j}
$$

Remark. Using the Lemma10 below, given $r>0$, we can always choose $z, \sigma_{0}$ and $\max _{k}\left|t_{k}\right|$ sufficiently small then $\max \left\{\left\|_{f} K\right\|_{0},\left\|_{0} K\right\|_{0}\right\} \leq r$. Now we claim that $\left\|_{f} K_{j}^{N}\right\|_{j}$ has the same bound as the $\left\|_{0} K_{j}^{N}\right\|_{j}$ in the last theorem.

Supposed that at $j=k$, we have: $\left\|_{f} K_{j}^{N}\right\|_{j} \leq r 2^{-k}$. As in the proof of Theorem 7 in (Dimock, [5]), we can say that $\mathcal{L}$ and $\left(\mathcal{L}_{1}+\mathcal{L}_{2}\right)$ is a contraction with norm less than $1 / 8$ and ${ }_{f} g\left(\sigma_{j},{ }_{f} K_{j}^{N},{ }_{0} K_{j}\right)$ is second order. Hence there are some constant $H$ such that: $\left\|_{f} g\left(\sigma_{j},{ }_{f} K_{j}^{N},{ }_{0} K_{j}^{N}\right)\right\| \leq H\left(\left|\sigma_{j}\right|^{2}+\right.$ $\left.\left\|_{0} K_{j}^{N}\right\|_{j}^{2}+\left\|_{f} K_{j}^{N}\right\|_{j}^{2}\right)$ with $\left|\sigma_{j}\right|,\left\|_{0} K_{j}^{N}\right\|_{j},\left\|_{f} K_{j}^{N}\right\|_{j}$ small. Then we have:

$$
\begin{aligned}
\left\|_{f} K_{j+1}^{N}\right\|_{j+1} & \leq \frac{1}{8}\left(\left\|_{0} K_{j}^{N}\right\|_{j}+\left\|\delta_{f} K_{j}^{N}\right\|_{j}\right)+H\left(\left|\sigma_{j}\right|^{2}+\left\|_{0} K_{j}^{N}\right\|_{j}^{2}+\left\|_{f} K_{j}^{N}\right\|_{j}^{2}\right) \\
& \leq \frac{1}{8}\left(2\left\|_{0} K_{j}^{N}\right\|_{j}+\left\|_{f} K_{j}^{N}\right\|_{j}\right)+3 H\left(r 2^{-j}\right)^{2} \\
& \leq \frac{1}{8}\left(3 r 2^{-j}\right)+3 H\left(r 2^{-j}\right)^{2} \\
& \leq r 2^{-j-1}
\end{aligned}
$$

for $r$ sufficiently small

The bound for $\left\|E^{N}\right\|_{j+1}$ comes from the bound on $\sigma_{j},\left\|_{f} K_{j}^{N}\right\|_{j}$, (125) and $A>1$.

Combining with the last theorem, for all $0 \leq j \leq N$ we can have:

$$
\left|\sigma_{j}\right| \leq r 2^{-j} \quad\left\|{ }_{f} K_{j}^{N}\right\|_{j} \leq r 2^{-j}
$$

and the extracted energies satisfy

$$
\left\|_{f} E_{j+1}^{N}\right\|_{j+1} \leq 2 \mathcal{C}\left(L^{d}\right) r 2^{-j}
$$

\section{The dipole gas}

\subsection{The initial density}

Now we consider the generating function:

$$
{ }_{f} Z_{N}(z, \sigma)=\int e^{i f(\phi)} \exp \left(z W\left(\Lambda_{N}, \sqrt{1+\sigma} \phi\right)-\sigma V\left(\Lambda_{N}, \phi\right)\right) d \mu_{C_{0}}(\phi)
$$

When $f=0$, it becomes

$$
{ }_{0} Z_{N}(z, \sigma)=\int \exp \left(z W\left(\Lambda_{N}, \sqrt{1+\sigma} \phi\right)-\sigma V\left(\Lambda_{N}, \phi\right)\right) d \mu_{C_{0}}(\phi)
$$


For $B \in \mathcal{B}_{0}$, we define: $W_{0}(B)=z W\left(\sqrt{1+\sigma_{0}}, B\right)$ as in (42) and $V_{0}(B)=\sigma_{0} V(B)$ as in (36). Then we follow with a Mayer expansion to put the density in the form we want.

$$
\begin{aligned}
{ }_{f} \mathcal{Z}_{0}^{N} & =\prod_{B \subset \Lambda_{N}} e^{i f(\phi)+W_{0}(B)-V_{0}(B)} \\
& =\prod_{B \subset \Lambda_{N}}\left(e^{-V_{0}(B)}+\left(e^{i f(\phi)+W_{0}(B)}-1\right) e^{-V_{0}(B)}\right) \\
& =\sum_{X \subset \Lambda_{N}} I_{0}\left(\sigma_{0}, \Lambda_{N}-X\right)_{f} K_{0}(X) \\
& =\left({ }_{f} I_{0}\left(\sigma_{0}\right) \circ{ }_{f} K_{0}\right)\left(\Lambda_{N}\right)
\end{aligned}
$$

where $I_{0}\left(\sigma_{0}, B\right)=e^{-V_{0}(B)}$ and ${ }_{f} K_{0}(X)={ }_{f} K_{0}\left(z, \sigma_{0}, X\right)$ is given by

$$
{ }_{f} K_{0}(X)=\prod_{B \subset X}\left(e^{\left.i f(\phi)\right|_{B}+W_{0}(B)}-1\right) e^{-V_{0}(B)}
$$

when $f(\phi)=\sum_{k=1}^{m} t_{k} \exp \left(i \partial_{\mu_{k}} \phi\left(x_{k}\right)\right)$, if $\left.(\phi)\right|_{B}=t_{k} \exp \left(i \partial_{\mu_{k}} \phi\left(x_{k}\right)\right)$ if $B=\left\{x_{k}\right\}$ for some $k$, otherwise $\left.\operatorname{if}(\phi)\right|_{B}=0$

or

$$
{ }_{f} K_{0}(X)=\prod_{B \subset X}\left(e^{\left.i f(\phi)\right|_{B}+W_{0}(B)}-1\right) e^{-V_{0}(B)}
$$

when $f(\phi)=f(\phi)=\sum_{k=1}^{m} t_{k} \partial_{\mu_{k}} \phi\left(x_{k}\right)$, if $\left.(\phi)\right|_{B}=t_{k} \partial_{\mu_{k}} \phi\left(x_{k}\right)$ if $B=\left\{x_{k}\right\}$ for some $k$, otherwise $\left.i f(\phi)\right|_{B}=0$

or

$$
{ }_{f} K_{0}(X)=\prod_{B \subset X}\left(e^{W_{0}(B)}-1\right) e^{-V_{0}(B)}
$$

when $f(\phi)=0$

Note that, when $f=0,{ }_{0} K_{0}$ actually is the $K_{0}$ in lemma 12, 5]. We also can prove the same result for ${ }_{f} K_{0}$.

Lemma 10 Given $1>r>0$, there are some sufficiently small $a(r), b(r)$ and $c(r)$ such that if $\max _{k}\left|t_{k}\right| \leq a(r),|z| \leq b(r)$ and $\left|\sigma_{0}\right| \leq c(r)$ then $\left\|_{f} K_{0}\left(z, \sigma_{0}\right)\right\|_{0} \leq r$. Furthermore ${ }_{f} K_{0}$ is a smooth function of $\left(z, \sigma_{0}\right)$, and analytic in $t_{k}$ for all $k=1, \ldots, m$.

Proof.

*When $f=0$, using lemma 12 [5], we have some $b_{0}(r), c_{0}(r)$ such that $\left\|_{0} K_{0}\left(z, \sigma_{0}\right)\right\|_{0} \leq r$ if $|z| \leq b_{0}(r)$ and $\left|\sigma_{0}\right| \leq c_{0}(r)$

*In the case $f(\phi)=\sum_{k=1}^{m} t_{k} \partial_{\mu_{k}} \phi\left(x_{k}\right)$, using ([5], (95)), for $\phi=\phi^{\prime}+\zeta$, we have:

$$
\begin{aligned}
& \left\|\left(e^{\left.i f(\phi)\right|_{B}+W_{0}(B)}-1\right)\right\|_{0}=\left\|\left(e^{\left.i f(\phi)\right|_{B}+z W\left(\sqrt{1+\sigma_{0}}, B\right)}-1\right)\right\|_{0} \\
& \leq \sum_{n=1}^{\infty} \frac{1}{n !}\left\|z W\left(\sqrt{1+\sigma_{0}}, B\right)+\left.i f(\phi)\right|_{B}\right\|_{0}^{n} \\
& \leq \sum_{n=1}^{\infty} \frac{1}{n !}\left(\left\|z W\left(\sqrt{1+\sigma_{0}}, B\right)\right\|_{0}+\left\|\left.i f(\phi)\right|_{B}\right\|_{0}\right)^{n} \\
& \leq \sum_{n=1}^{\infty} \frac{1}{n !}\left(2|z| e^{h \sqrt{d\left(1+\sigma_{0}\right)}}+\max _{k}\left|t_{k}\right| h^{-1}\|\phi\|_{\Phi_{0}\left(B^{*}\right)}\right)^{n}
\end{aligned}
$$


We can assume that $\max _{k}\left|t_{k}\right| h^{-1} \leq 1$. Applying lemma 3 in [5], we get $\left\|e^{-V_{0}(B)}\right\|_{s, 0} \leq 2$.

$$
\begin{aligned}
& \left\|_{f} K_{0}(B)\right\|_{0}=\sup _{\phi^{\prime}, \zeta}\left\|_{f} K_{0}\left(B, \phi^{\prime}+\zeta\right)\right\|_{0} G_{0}\left(X, \phi^{\prime}, \zeta\right)^{-1} \\
& \leq \sup _{\phi^{\prime}, \zeta}\left\|\left(e^{\left.i f(\phi)\right|_{B}+W_{0}(B)}-1\right)\right\|_{0}\left\|e^{-V_{0}(B)}\right\|_{0} G_{s, 0}\left(X, \phi^{\prime}, \zeta\right)^{-2} \\
& \leq\left\|e^{-V_{0}(B)}\right\|_{s, 0} \sup _{\phi^{\prime}, \zeta}\left\|\left(e^{\left.i f(\phi)\right|_{B}+W_{0}(B)}-1\right)\right\|_{0} G_{s, 0}\left(X, \phi^{\prime}, \zeta\right)^{-1} \\
& \leq 2 \sup _{\phi^{\prime}, \zeta}\left(\exp \left(2|z| e^{h \sqrt{d\left(1+\sigma_{0}\right)}}+\max _{k}\left|t_{k}\right| h^{-1}\left\|\phi^{\prime}+\zeta\right\|_{\Phi_{0}\left(B^{*}\right)}\right)-1\right) G_{s, 0}\left(X, \phi^{\prime}, \zeta\right)^{-1} \\
& \leq 2 \sup _{\phi^{\prime}, \zeta}\left(\exp \left(2|z| e^{h \sqrt{d\left(1+\sigma_{0}\right)}}\right)-1\right) \exp \left(\max _{k}\left|t_{k}\right| h^{-1}\left\|\phi^{\prime}+\zeta\right\|_{\Phi_{0}\left(B^{*}\right)}\right) G_{s, 0}\left(X, \phi^{\prime}, \zeta\right)^{-1} \\
& +2 \sup _{\phi^{\prime}, \zeta}\left(\exp \left(\max _{k}\left|t_{k}\right| h^{-1}\left\|\phi^{\prime}+\zeta\right\|_{\Phi_{0}\left(B^{*}\right)}\right)-1\right) G_{s, 0}\left(X, \phi^{\prime}, \zeta\right)^{-1} \\
& \leq 2 \sup _{\phi^{\prime}, \zeta}\left(\exp \left(2|z| e^{h \sqrt{d\left(1+\sigma_{0}\right)}}\right)-1\right) \exp \left(\left\|\phi^{\prime}+\zeta\right\|_{\Phi_{0}\left(B^{*}\right)}\right) e^{-\left\|\phi^{\prime}\right\|_{\Phi_{0}\left(B^{*}\right)}^{2}-\|\zeta\|_{\Phi_{0}\left(B^{*}\right)}^{2}} \\
& +2 \sup _{\phi^{\prime}, \zeta}\left(\exp \left(\max _{k}\left|t_{k}\right| h^{-1}\left\|\phi^{\prime}+\zeta\right\|_{\Phi_{0}\left(B^{*}\right)}\right)-1\right) e^{-\left\|\phi^{\prime}\right\|_{\Phi_{0}\left(B^{*}\right)}^{2}-\|\zeta\|_{\Phi_{0}\left(B^{*}\right)}^{2}}
\end{aligned}
$$

Because $\exp \left(\left\|\phi^{\prime}+\zeta\right\|_{\Phi_{0}\left(B^{*}\right)}\right) \exp \left(-\left\|\phi^{\prime}\right\|_{\Phi_{0}\left(B^{*}\right)}^{2}-\|\zeta\|_{\Phi_{0}\left(B^{*}\right)}^{2}\right)$ is bounded and

$$
\lim _{z, \sigma_{0} \rightarrow 0}\left(\exp \left(2|z| e^{h \sqrt{d\left(1+\sigma_{0}\right)}}\right)-1\right)=0
$$

there exist some sufficiently small $b_{1}(r), c_{1}(r)>0$ such that we have

$$
2 \sup _{\phi^{\prime}, \zeta}\left(\exp \left(2|z| e^{h \sqrt{d\left(1+\sigma_{0}\right)}}\right)-1\right) \exp \left(\left\|\phi^{\prime}+\zeta\right\|_{\Phi_{0}\left(B^{*}\right)}\right) e^{-\left\|\phi^{\prime}\right\|_{\Phi_{0}\left(B^{*}\right)}^{2}-\|\zeta\|_{\Phi_{0}\left(B^{*}\right)}^{2}} \leq \frac{r}{4 A}
$$

for all $|z| \leq b_{1}(r)$ and $\left|\sigma_{0}\right| \leq c_{1}(r)$.

For other part, we have:

$$
\begin{aligned}
& 2 \sup _{\phi^{\prime}, \zeta}\left(\exp \left(\max _{k}\left|t_{k}\right| h^{-1}\left\|\phi^{\prime}+\zeta\right\|_{\Phi_{0}\left(B^{*}\right)}\right)-1\right) \exp \left(-\left\|\phi^{\prime}\right\|_{\Phi_{0}\left(B^{*}\right)}^{2}-\|\zeta\|_{\Phi_{0}\left(B^{*}\right)}^{2}\right) \\
& \leq 2 \sup _{\phi^{\prime}, \zeta}\left(\exp \left(\left\|\phi^{\prime}\right\|_{\Phi_{0}\left(B^{*}\right)}+\|\zeta\|_{\Phi_{0}\left(B^{*}\right)}\right)-1\right) \exp \left(-\left\|\phi^{\prime}\right\|_{\Phi_{0}\left(B^{*}\right)}^{2}-\|\zeta\|_{\Phi_{0}\left(B^{*}\right)}^{2}\right)
\end{aligned}
$$

We also can find some sufficiently large $H$ such that if $\left\|\phi^{\prime}\right\|_{\Phi_{0}\left(B^{*}\right)}+\|\zeta\|_{\Phi_{0}\left(B^{*}\right)} \geq H$ then

$$
2\left(\exp \left(\left\|\phi^{\prime}\right\|_{\Phi_{0}\left(B^{*}\right)}+\|\zeta\|_{\Phi_{0}\left(B^{*}\right)}\right)-1\right) \exp \left(-\left\|\phi^{\prime}\right\|_{\Phi_{0}\left(B^{*}\right)}^{2}-\|\zeta\|_{\Phi_{0}\left(B^{*}\right)}^{2}\right) \leq \frac{r}{4 A}
$$

For $\left\|\phi^{\prime}\right\|_{\Phi_{0}\left(B^{*}\right)}+\|\zeta\|_{\Phi_{0}\left(B^{*}\right)} \leq H$, we have $\left\|\phi^{\prime}+\zeta\right\|_{\Phi_{0}\left(B^{*}\right)} \leq\left\|\phi^{\prime}\right\|_{\Phi_{0}\left(B^{*}\right)}+\|\zeta\|_{\Phi_{0}\left(B^{*}\right)} \leq H$. So with $\max _{k}\left|t_{k}\right| \leq a_{1}(r)$ sufficiently small and $\left\|\phi^{\prime}\right\|_{\Phi_{0}\left(B^{*}\right)}+\|\zeta\|_{\Phi_{0}\left(B^{*}\right)} \leq H$,

$$
2\left(\exp \left(\max _{k}\left|t_{k}\right| h^{-1}\left\|\phi^{\prime}+\zeta\right\|_{\Phi_{0}\left(B^{*}\right)}\right)-1\right) \exp \left(-\left\|\phi^{\prime}\right\|_{\Phi_{0}\left(B^{*}\right)}^{2}-\|\zeta\|_{\Phi_{0}\left(B^{*}\right)}^{2}\right) \leq \frac{r}{4 A}
$$

In summary we can always choose sufficiently small $a(r), b(r), c(r)$ such that if $\max _{k}\left|t_{k}\right| \leq a_{1}(r)$, $|z| \leq b_{1}(r)$, and $\left|\sigma_{0}\right| \leq c_{1}(r)$ then

$$
\left\|_{f} K_{0}(B)\right\|_{0} \leq 2 \frac{r}{4 A}=\frac{r}{2 A} \quad \forall B \in \mathcal{B}_{0}
$$


For those $a_{1}(r), b_{1}(r), c_{1}(r), \max _{k}\left|t_{k}\right| \leq a_{1}(r),|z| \leq b_{1}(r)$, and $\left|\sigma_{0}\right| \leq c_{1}(r)$ we have

$$
\begin{aligned}
\left\|_{f} K_{0}\right\|_{0} & =\sup _{X \in \mathcal{P}_{0, c}}\left\|_{f} K_{0}(X)\right\|_{0} A^{|X|_{0}} \\
& \leq \sup _{X \in \mathcal{P}_{0, c}}\left(\prod_{B \subset X}\left\|_{f} K_{0}(B)\right\|_{0}\right) A^{|X|_{0}} \\
& \leq \sup _{X \in \mathcal{P}_{0, c}}\left(\frac{r}{2 A}\right)^{|X|_{0}} A^{|X|_{0}} \leq \frac{r}{2}<r
\end{aligned}
$$

* In the last case, $f(\phi)=\sum_{k=1}^{m} t_{k} \exp \left(i \partial_{\mu_{k}} \phi\left(x_{k}\right)\right)$, we have:

$$
\begin{aligned}
\left\|\left(e^{\left.i f(\phi)\right|_{B}+W_{0}(B)}-1\right)\right\|_{0} & \leq \sum_{n=1}^{\infty} \frac{1}{n !}\left(2|z| e^{h \sqrt{d\left(1+\sigma_{0}\right)}}+\max _{k}\left|t_{k}\right|\right)^{n} \\
& =\exp \left(2|z| e^{h \sqrt{d\left(1+\sigma_{0}\right)}}+\max _{k}\left|t_{k}\right|\right)-1
\end{aligned}
$$

Using the same argument as above, we can choose some sufficiently small $a_{2}(r), b_{2}(r), c_{2}(r)$ such that $\left\|_{f} K_{0}\left(z, \sigma_{0}\right)\right\|_{0} \leq r$ when $\max _{k}\left|t_{k}\right| \leq a_{2}(r),|z| \leq b_{2}(r)$ and $\left.\left|\sigma_{0}\right| \leq c_{(} r\right)$.

Now we just simply pick $a(r)=\max \left\{a_{1}(r), a_{2}(r)\right\}, b(r)=\max \left\{b_{0}(r), b_{1}(r), b_{2}(r)\right\}$ and $c(r)=$ $\max \left\{c_{0}(r), c_{1}(r), c_{2}(r)\right\}$.

The smoothness follows similarly from Lemma 12, (Dimock, 5 ). 7

Remark. We have ${ }_{f} K_{0}$ is analytic. For each step when we jump from $j$-scale to $(j+1)$-scale, the analyticity of ${ }_{f} K$ still holds for the next scale.

Noticing that ${ }_{0} K_{0}$ is just the $K_{0}$ in Section 6, (Dimock, [5]), we need the following lemma to apply Theorem 8

Lemma 11 (Lemma 13, [5])

The equation $\sigma=h\left({ }_{0} K_{0}(z, \sigma)\right)$ defines a smooth implicit function $\sigma=\sigma(z)$ near the origin which satisfies $\sigma(0)=0$. have

Taking $|z|$ sufficiently small and choosing $\sigma_{0}=\sigma(z)$, we can apply theorem 8 , For $0 \leq l \leq N$, we

$$
{ }_{f} Z_{N}=\exp \left(\sum_{j=1}^{l} \sum_{B \in \mathcal{B}_{j}\left(\Lambda_{N}\right)}{ }_{f} E_{j}^{N}(B)\right) \int\left({ }_{f} I_{l}\left(\sigma_{l}\right) \circ{ }_{f} K_{l}^{N}\right)\left(\Lambda_{N}\right) d \mu_{C_{l}}
$$

where $\left|\sigma_{j}\right| \leq r 2^{-j}$ and $\left\|_{f} K_{j}^{N}\right\|_{j} \leq r 2^{-j}$ and $\left\|_{f} E_{j+1}^{N}\right\|_{j+1} \leq \mathcal{O}\left(L^{d}\right) r 2^{-j}$.

\subsection{Completing the proof of Theorem 1}

Theorem 9 For $|z|$ and $\max _{k}\left|t_{k}\right|$ sufficiently small the following limit exists:

$$
\lim _{N \rightarrow \infty}\left|\Lambda_{N}\right|^{-1} \log { }_{f} Z_{N}^{\prime}(z, \sigma(z))
$$

${ }^{7}$ Instead of using the usual estimates, such as $\left(1+\|\phi\|_{\Phi_{j}\left(B^{*}\right)}^{2}\right) \leq \exp \left(\|\phi\|_{\Phi_{j}\left(B^{*}\right)}^{2}\right)=G_{s, j}(B, \phi, 0)$, we can use

$$
\left(1+\|\phi\|_{\Phi_{j}\left(B^{*}\right)}^{2}\right)=k\left(\frac{1}{k}+\frac{1}{k}\|\phi\|_{\Phi_{j}\left(B^{*}\right)}^{2}\right) \leq k \exp \left(\frac{1}{k}\|\phi\|_{\Phi_{j}\left(B^{*}\right)}^{2}\right)=k G_{s, j}^{\frac{1}{k}}(B, \phi, 0)
$$

for any positive integer $k$, and so forth 


\section{Proof.}

With updated index, the proof can go exactly the same as the proof of Theorem 8, 5]. We take $l=N$ in (150). At this scale there is only one block $\Lambda_{N} \in \mathcal{B}_{N}\left(\Lambda_{N}\right)$ and so we have

$$
\begin{aligned}
\left|\Lambda_{N}\right|^{-1} \log { }_{f} Z_{N}^{\prime}(z, \sigma(z)) & =\left|\Lambda_{N}\right|^{-1} \sum_{j=1}^{N} \sum_{B \in \mathcal{B}_{j}\left(\Lambda_{N}\right)}{ }_{f} E_{j}^{N}(B) \\
& +\left|\Lambda_{N}\right|^{-1} \log \left(\int\left[{ }_{f} I_{N}\left(\sigma_{N}, \Lambda_{N}\right)+{ }_{f} K_{N}^{N}\left(\Lambda_{N}\right)\right] d \mu_{C_{N}}\right)
\end{aligned}
$$

The second term has the form

$$
\left|\Lambda_{N}\right|^{-1} \log \left(1+\int{ }_{f} F_{N} d \mu_{C_{N}}\right)
$$

where

$$
\begin{gathered}
{ }_{f} T_{N}=\left(1+\int{ }_{f} F_{N} d \mu_{C_{N}}\right) \\
{ }_{f} F_{N}\left(\Lambda_{N}\right)={ }_{f} F_{N}={ }_{f} I_{N}\left(\sigma_{N}, \Lambda_{N}\right)-1+{ }_{f} K_{N}^{N}\left(\Lambda_{N}\right)
\end{gathered}
$$

By (75) and (126) in [5], we have

$$
\begin{aligned}
\left\|_{f} I_{N}\left(\sigma_{N}, \Lambda_{N}\right)-1\right\|_{N} & \leq 4 c^{-1} h^{2}\left|\sigma_{N}\right| \leq 4 c^{-1} h^{2} r 2^{-N} \\
\left\|_{f} K_{N}^{N}\left(\Lambda_{N}\right)\right\|_{N} & \leq A^{-1}\left\|_{f} K_{N}^{N}\right\|_{N} \leq A^{-1} r 2^{-N}
\end{aligned}
$$

so that $\left\|_{f} F_{N}\left(\Lambda_{N}\right)\right\|_{N} \leq\left(4 c^{-1} h^{2}+A^{-1}\right) r\left(2^{-N}\right)$ which is $\mathcal{O}\left(2^{-N}\right)$ as $N \rightarrow \infty$.

In lemma 14 [5]) Dimock has proved that for $h$ sufficiently large

$$
\int G_{N}\left(\Lambda_{N}, 0, \zeta\right) d \mu_{C_{N}}(\zeta) \leq 2
$$

Then we estimate

$$
\begin{aligned}
\left|\int{ }_{f} F_{N}\left(\Lambda_{N}\right) d \mu_{C_{N}}\right| & \leq\left\|_{f} F_{N}\left(\Lambda_{N}\right)\right\|_{N} \int G_{N}\left(\Lambda_{N}, 0, \zeta\right) d \mu_{C_{N}}(\zeta) \\
& \leq 2\left\|F\left(\Lambda_{N}\right)\right\|_{N} \\
& \leq 2\left(4 c^{-1} h^{2}+A^{-1}\right) r\left(2^{-N}\right)
\end{aligned}
$$

Hence the expression (153) is $\mathcal{O}\left(2^{-N}\right)\left|\Lambda_{N}\right|^{-1}$ and goes to zero very quickly as $N \rightarrow \infty$

The rest of the proof came as in the proof of Theorem 8 in [5].

\section{Correlation functions: estimates and infinite volume limit}

Note: We always can assume that $L \gg 2^{d+3}+1$

6.1 In the case: $f(\phi)=\sum_{k=1}^{m} t_{k} \partial_{\mu_{k}} \phi\left(x_{k}\right)$

For $x_{k} \in \mathbb{Z}^{d}$ are different points; $\mu_{k} \in\{ \pm 1, \ldots, \pm d\}$ and $t_{k}$ complex and $\left|t_{k}\right| \leq a=a(r)$ for $\forall k$ : $1,2, \ldots m$. 


\subsubsection{Proof of Theorem 2}

Using (150) with $l=N$, for the truncated correlation functions, we have:

$$
\begin{aligned}
& \left.\mathcal{G}^{t}\left(x_{1}, x_{2}, \ldots x_{m}\right) \equiv\left\langle\prod_{k=1}^{m} \partial_{\mu_{k}} \phi\left(x_{k}\right)\right\rangle^{t} \equiv i^{m} \frac{\partial^{m}}{\partial t_{1} \ldots \partial t_{m}} \log _{f} Z^{\prime}\right|_{t_{1}=0, \ldots t_{m}=0} \\
& =\left.i^{m} \frac{\partial^{m}}{\partial t_{1} \ldots \partial t_{m}}\left(\sum_{j=1}^{N} \sum_{B \in \mathcal{B}_{j}\left(\Lambda_{N}\right)}{ }_{f} E_{j}^{N}(B)\right)\right|_{t_{1}=0, \ldots t_{m}=0} \\
& \quad+\left.i^{m} \frac{\partial^{m}}{\partial t_{1} \ldots \partial t_{m}} \log \int\left({ }_{f} I_{N}\left(\sigma_{N}\right) \circ{ }_{f} K_{N}^{N}\right)\left(\Lambda_{N}\right) d \mu_{C_{N}}\right|_{t_{1}=0, \ldots t_{m}=0} \\
& =\left.i^{m} \sum_{j=1}^{N} \sum_{B \in \mathcal{B}_{j}\left(\Lambda_{N}\right)} \frac{\partial^{m}}{\partial t_{1} \ldots \partial t_{m}} E_{j}^{N}(B)\right|_{t_{1}=0, \ldots t_{m}=0} \\
& \quad+\left.i^{m} \frac{\partial^{m}}{\partial t_{1} \ldots \partial t_{m}} \log \left(1+\int\left({ }_{f} I_{N}\left(\sigma_{N}\right)-1+{ }_{f} K_{N}^{N}\right)\left(\Lambda_{N}\right) d \mu_{C_{N}}\right)\right|_{t_{1}=0, \ldots t_{m}=0}
\end{aligned}
$$

Now we consider the quantity:

$$
\begin{aligned}
{ }_{f} F_{N} & \left.\equiv \sum_{j=1}^{N} \sum_{B \in \mathcal{B}_{j}\left(\Lambda_{N}\right)} \frac{\partial^{m}}{\partial t_{1} \ldots \partial t_{m}}{ }_{f} E_{j}^{N}(B)\right|_{t_{1}=0, \ldots t_{m}=0} \\
& =\left.\sum_{j=0}^{N-1} \sum_{B \in \mathcal{B}_{j}\left(\Lambda_{N}\right)} \frac{\partial^{m}}{\partial t_{1} \ldots \partial t_{m}}{ }_{f} \beta\left({ }_{f} K_{j}^{N}, B\right)\right|_{t_{1}=0, \ldots t_{m}=0} \\
& =\left.\sum_{j=0}^{N-1} \sum_{B \in \mathcal{B}_{j}\left(\Lambda_{N}\right)} \frac{\partial^{m}}{\partial t_{1} \ldots \partial t_{m}} \sum_{X \in \mathcal{S}_{j}, X \supset B} \frac{1}{|X|_{j}}{ }^{f} K_{j}^{N \#}(X, 0)\right|_{t_{1}=0, \ldots t_{m}=0}
\end{aligned}
$$

by the definition of ${ }_{f} \beta$ in (89).

We notice that $\frac{\partial^{m}}{\partial t_{1} \ldots \partial t_{m}} f E_{j}(B)=0$ unless $B^{*} \supset\left\{x_{1}, x_{2}, \ldots x_{m}\right\}$. Therefore,

$$
{ }_{f} F_{N}=\left.\sum_{j=0}^{N-1} \sum_{\substack{B \in \mathcal{B}_{j}\left(\Lambda_{N}\right) \\ B^{*} \supset\left\{x_{1}, x_{2}, \ldots x_{m}\right\}}} \frac{\partial^{m}}{\partial t_{1} \ldots \partial t_{m}} \sum_{X \in \mathcal{S}_{j}, X \supset B} \frac{1}{|X|_{j}} f_{j} K_{j}^{N \#}(X, 0)\right|_{t_{1}=0, \ldots t_{m}=0}
$$

Note: Let $\eta=\min \{d / 2,2\}$. For any small $\epsilon>0$, we can always find $A, L$ sufficiently large such that:

$$
\begin{gathered}
\left\|\left(\mathcal{L}_{1}+\mathcal{L}_{2}+\mathcal{L}_{3}^{\prime}+\mathcal{L}_{4}\right)\left({ }_{0} K\right)\right\|_{j+1} \leq \frac{1}{4 L^{\eta-\epsilon}}\left\|_{0} K\right\|_{j} \\
\left\|\left(\mathcal{L}_{1}+\mathcal{L}_{2}\right)\left(\delta_{f} K\right)\right\|_{j+1} \leq \frac{1}{4 L^{\eta-\epsilon}}\left(\left\|\delta_{f} K\right\|_{j}\right) \leq \frac{1}{4 L^{\eta-\epsilon}}\left(\|\|_{0} K\left\|_{j}+\right\|{ }_{f} K \|_{j}\right)
\end{gathered}
$$

with $j \geq 1$ by using the explicit upper bounds in Lemmas 3 4, 6, and 8 ,

Then we can replace $\mu=1 / 2$ in Theorem 7 by $\mu=1 / M$ for $M=L^{\eta-\epsilon} \geq 2$. We still have $\left|\sigma_{j}\right| \leq r M^{-j}$ and $\left\|_{f} K_{j}^{N}\right\|_{j} \leq r M^{-j}$ and $\left\|_{f} E_{j+1}^{N}\right\|_{j+1} \leq \mathcal{O}\left(L^{d}\right) r M^{-j}$ with $\max _{k}\left|t_{k}\right|<a$ sufficiently small and $0 \leq j \leq N-1$. Because ${ }_{f} K_{j}^{N \#}(X, 0)$ is analytic, using Cauchy's bound and (79) in [5], we have:

$$
\begin{aligned}
\left|\frac{\partial^{m}}{\partial t_{1} \ldots \partial t_{m}}{ }_{f} K_{j}^{N \#}(X, 0)\right|_{t_{1}=0, \ldots t_{m}=0} \mid & \leq \frac{m !}{a^{m}}\left(\frac{A}{2}\right)^{-|X|_{j}}\left\|_{f} K_{j}^{N}\right\|_{j} \\
& \leq \frac{m !}{a^{m}}\left(\frac{A}{2}\right)^{-|X|_{j}} r M^{-j}
\end{aligned}
$$


Then

$$
\begin{aligned}
& \left|\frac{\partial^{m}}{\partial t_{1} \ldots \partial_{t_{m}}} \sum_{X \in \mathcal{S}_{j}, X \supset B} \frac{1}{|X|_{j}}{ }_{f} K_{j}^{N \#}(X, 0)\right|_{t_{1}=0, \ldots t_{m}=0} \mid \\
& \leq \sum_{X \in \mathcal{S}_{j}, X \supset B} \frac{1}{|X|_{j}}\left|\frac{\partial^{m}}{\partial t_{1} \ldots \partial t_{m}}{ }_{f} K_{j}^{N \#}(X, 0)\right|_{t_{1}=0, \ldots t_{m}=0} \mid \\
& \leq \sum_{X \in \mathcal{S}_{j}, X \supset B} \frac{1}{|X|_{j}} \frac{m !}{a^{m}}\left(\frac{A}{2}\right)^{-|X|_{j}} r M^{-j} \\
& \leq n_{3}\left(d, \frac{A}{2}\right) \frac{m ! r M^{-j}}{a^{m}}
\end{aligned}
$$

So

$$
\left|F_{N}\right| \leq \sum_{j=0}^{N-1} \sum_{\substack{B \in \mathcal{B}_{j}\left(\Lambda_{N}\right) \\ B^{*} \supset\left\{x_{1}, x_{2}, \ldots x_{m}\right\}}} n_{3}\left(d, \frac{A}{2}\right) \frac{m ! r M^{-j}}{a^{m}}
$$

By (153)-(157), we have:

$$
\begin{aligned}
& \left|\log \left(1+\int\left({ }_{f} I_{N}\left(\sigma_{N}\right)-1+{ }_{f} K_{N}^{N}\right)\left(\Lambda_{N}\right) d \mu_{C_{N}}\right)\right| \\
& \leq \log \left(1+2\left\|F\left(\Lambda_{N}\right)\right\|_{N}\right) \\
& \leq \log \left(1+2\left[4 c^{-1} h^{2}+A^{-1}\right] r 2^{-N}\right)
\end{aligned}
$$

Using the Cauchy's bound as above, we obtain:

$$
\begin{aligned}
& \left|\frac{\partial^{m}}{\partial t_{1} \ldots \partial t_{m}} \log \left(1+\int\left({ }_{f} I_{N}\left(\sigma_{N}\right)-1+{ }_{f} K_{N}^{N}\right)\left(\Lambda_{N}\right) d \mu_{C_{N}}\right)\right|_{t_{1}=0, \ldots t_{m}=0} \mid \\
& \leq \frac{m !}{a^{m}} \log \left(1+2\left[4 c^{-1} h^{2}+A^{-1}\right] r 2^{-N}\right)
\end{aligned}
$$

So

$$
\lim _{N \rightarrow \infty}\left|\frac{\partial^{m}}{\partial t_{1} \ldots \partial t_{m}} \log \left(1+\int\left({ }_{f} I_{N}\left(\sigma_{N}\right)-1+{ }_{f} K_{N}^{N}\right)\left(\Lambda_{N}\right) d \mu_{C_{N}}\right)\right|_{t_{1}=0, \ldots t_{m}=0} \mid=0
$$

Now let $j_{0}$ be the smallest integer such that $\exists B \in \mathcal{B}_{j_{0}}: B^{*} \supset\left\{x_{1}, x_{2}, \ldots x_{m}\right\}$.

Without loosing the generality, we can assume that $\left|x_{1}-x_{2}\right|=\operatorname{diam}\left(x_{1}, \ldots x_{m}\right)$

For every $j \geq j_{0}$, let $B_{j}^{1} \in \mathcal{B}_{j}$ be the unique $j$-block that contains $\left\{x_{1}\right\}$. For any $B \in \mathcal{B}_{j}, j \geq j_{0}$ with $B^{*} \supset\left\{x_{1}, x_{2}, \ldots x_{m}\right\}, B$ must be in $B_{j}^{1^{*}}$.

We have

$$
\begin{aligned}
\left|{ }_{f} F_{N}\right| & \leq \sum_{j=0}^{N-1} \sum_{\substack{B \in \mathcal{B}_{j}\left(\Lambda_{N}\right) \\
B^{*} \supset\left\{x_{1}, x_{2}, \ldots x_{m}\right\}}} n_{3}\left(d, \frac{A}{2}\right) \frac{m ! r M^{-j}}{a^{m}} \\
& =\sum_{j=j_{0}}^{N-1} \sum_{\substack{B \in \mathcal{B}_{j}\left(\Lambda_{N}\right) \\
B^{*} \supset\left\{x_{1}, x_{2}, \ldots x_{m}\right\}}} n_{3}\left(d, \frac{A}{2}\right) \frac{m ! r M^{-j}}{a^{m}}
\end{aligned}
$$


Since $M \geq 2$, the last part of (168) is bounded by

$$
\begin{aligned}
\sum_{j=j_{0}}^{N-1} \sum_{\substack{B \in \mathcal{B}_{j}\left(\Lambda_{N}\right) \\
B^{*} \supset\left\{x_{1}, x_{2}, \ldots x_{m}\right\}}} n_{3}\left(d, \frac{A}{2}\right) \frac{m ! r M^{-j}}{a^{m}} & \leq \sum_{j=j_{0}}^{N-1} \sum_{\substack{B \in \mathcal{B}_{j}\left(\Lambda_{N}\right) \\
B \in B_{j}^{1 *}}} n_{3}\left(d, \frac{A}{2}\right) \frac{m ! r M^{-j}}{a^{m}} \\
& \leq \sum_{j=j_{0}}^{N-1}\left(2^{d} 2\right)^{d} n_{3}\left(d, \frac{A}{2}\right) \frac{m ! r M^{-j}}{a^{m}} \\
& \leq 2^{d(d+1)} n_{3}\left(d, \frac{A}{2}\right) 2 \frac{m ! r M^{-j_{0}}}{a^{m}}
\end{aligned}
$$

Therefore, we have:

$$
\left|{ }_{f} F_{N}\right| \leq 2^{d(d+1)} n_{3}\left(d, \frac{A}{2}\right) 2 \frac{m ! r M^{-j_{0}}}{a^{m}}
$$

By the definition of $j_{0}$, we have: $\left|x_{1}-x_{2}\right| \leq d 2^{d+1} L^{j_{0}}$. Because $M=L^{\eta-\epsilon}$, we get

$$
\begin{aligned}
M^{-j_{0}}=L^{-j_{0}(\eta-\epsilon)} & \leq\left(d 2^{d+1}\right)^{\eta}\left|x_{1}-x_{2}\right|^{-\eta+\epsilon} \\
& =\left(d 2^{d+1}\right)^{\eta} \operatorname{diam}^{-\eta+\epsilon}\left(x_{1}, \ldots, x_{m}\right)
\end{aligned}
$$

Hence, we have:

$$
\left|{ }_{f} F_{N}\right| \leq 2^{d(d+1)} n_{3}\left(d, \frac{A}{2}\right) 2 \frac{m ! r}{a^{m}} \operatorname{diam}^{-\eta+\epsilon}\left(x_{1}, \ldots x_{m}\right)\left(d^{\eta} 2^{\eta(d+1)}\right)
$$

Using this with (167), we obtain:

$$
\left|\frac{\partial^{m}}{\partial t_{1} \ldots \partial t_{m}} \log _{f} Z^{\prime}\right|_{t_{1}=0, \ldots t_{m}=0} \mid \leq 2^{d(d+1)} 4 n_{3}\left(d, \frac{A}{2}\right) \frac{m ! r}{a^{m}} \operatorname{diam}^{-\eta+\epsilon}\left(x_{1}, \ldots x_{m}\right)\left(d^{\eta} 2^{\eta(d+1)}\right)
$$

Combining with (31), we get $n_{3}\left(d, \frac{A}{2}\right) 2^{d(d+1)} 4 r\left(d 2^{d+1}\right)^{\eta} \leq 1$ with sufficiently large $A$. Therefore, with sufficiently large $A$, we have:

$$
\begin{aligned}
\left|\mathcal{G}^{t}\left(x_{1}, x_{2}, \ldots x_{m}\right)\right| & =\left|\left\langle\prod_{k=1}^{m} \partial_{\mu_{k}} \phi\left(x_{k}\right)\right\rangle^{t}\right|=\left|\frac{\partial^{m}}{\partial t_{1} \ldots \partial t_{m}} \log _{f} Z^{\prime}\right|_{t_{1}=0, \ldots t_{m}=0} \mid \\
& \leq \frac{m !}{a^{m}} \operatorname{diam}^{-\eta+\epsilon}\left(x_{1}, \ldots x_{m}\right)
\end{aligned}
$$

We complete the proof of Theorem 2 .

Remark. Actually for any $N-1 \geq q \geq j_{0}$, similarly to (169), we have

$$
\begin{aligned}
& \left|\sum_{j=q}^{N-1} \sum_{\substack{B \in \mathcal{B}_{j}\left(\Lambda_{N}\right) \\
B^{*} \supset\left\{x_{1}, x_{2}, \ldots x_{m}\right\}}} \frac{\partial^{m}}{\partial t_{1} \ldots \partial t_{m}} \sum_{X \in \mathcal{S}_{j}, X \supset B} \frac{1}{|X|_{j}} f_{j} K_{j}^{N \#}(X, 0)\right|_{t_{1}=0, \ldots t_{m}=0} \mid \\
& \leq \sum_{j=q}^{N-1} \sum_{\substack{B \in \mathcal{B}_{j}\left(\Lambda_{N}\right) \\
B^{*} \supset\left\{x_{1}, x_{2}, \ldots x_{m}\right\}}} n_{3}\left(d, \frac{A}{2}\right) \frac{m ! r M^{-j}}{a^{m}} \\
& \leq 2^{d(d+1)} n_{3}\left(d, \frac{A}{2}\right) 2 \frac{m ! r M^{-q}}{a^{m}}
\end{aligned}
$$




\subsubsection{Proof of Theorem 3}

Now we fix the set $\left\{x_{1}, x_{2}, \ldots x_{m}\right\}$. Let $j_{1}$ be the smallest integer such that $B_{j_{1}}^{0} \supset\left\{x_{1}, x_{2}, \ldots x_{m}\right\}$. Then $j_{1}$ is the smallest integer which is greater than $\log _{L} \max _{i}\left\|x_{i}\right\|_{\infty}$. We also have: $j_{0} \leq j_{1}$.

Let $q$ be any number such that $q \geq j_{1}+1 \geq j_{0}+1$. And let $N_{1}, N_{2}$ be any integers such that $N_{2} \geq N_{1}>q$. Using the definition of $j_{0}$, we have

$$
\begin{aligned}
{ }_{f} F_{N_{1}} & =\left.\sum_{j=j_{0}}^{q-1} \sum_{\substack{B \in \mathcal{B}_{j}\left(\Lambda_{N}\right) \\
B^{*} \supset\left\{x_{1}, x_{2}, \ldots x_{m}\right\}}} \frac{\partial^{m}}{\partial t_{1} \ldots \partial t_{m}} \sum_{X \in \mathcal{S}_{j}, X \supset B} \frac{1}{|X|_{j}} f K_{j}^{N_{2} \#}(X, 0)\right|_{t_{1}=0, \ldots t_{m}=0} \\
& +\left.\sum_{j=q}^{N_{1}-1} \sum_{\substack{B \in \mathcal{B}_{j}\left(\Lambda_{N}\right) \\
B^{*} \supset\left\{x_{1}, x_{2}, \ldots x_{m}\right\}}} \frac{\partial^{m}}{\partial t_{1} \ldots \partial t_{m}} \sum_{X \in \mathcal{S}_{j}, X \supset B} \frac{1}{|X|_{j}} f K_{j}^{N_{2} \#}(X, 0)\right|_{t_{1}=0, \ldots t_{m}=0} \\
{ }_{f} F_{N_{2}} & =\left.\sum_{j=j_{0}}^{q-1} \sum_{\substack{\left.B \in \mathcal{B}_{j}\left(\Lambda_{N}\right) \\
B^{*} \supset x_{1}, x_{2}, \ldots x_{m}\right\}}} \frac{\partial^{m}}{\partial t_{1} \ldots \partial t_{m}} \sum_{X \in \mathcal{S}_{j}, X \supset B} \frac{1}{|X|_{j}} f K_{j}^{N_{2} \#}(X, 0)\right|_{t_{1}=0, \ldots t_{m}=0} \\
& +\left.\sum_{j=q}^{N_{2}-1} \sum_{\substack{B \in \mathcal{B}_{j}\left(\Lambda_{N}\right) \\
B^{*} \supset\left\{x_{1}, x_{2}, \ldots x_{m}\right\}}} \frac{\partial^{m}}{\partial t_{1} \ldots \partial t_{m}} \sum_{X \in \mathcal{S}_{j}, X \supset B} \frac{1}{|X|_{j}} f_{j} K_{j}^{N_{2} \#}(X, 0)\right|_{t_{1}=0, \ldots t_{m}=0}
\end{aligned}
$$

We also notice that:

$$
\begin{aligned}
& \left.\sum_{j=j_{0}}^{q-1} \sum_{\substack{B \in \mathcal{B}_{j}\left(\Lambda_{N}\right) \\
B^{*} \supset\left\{x_{1}, x_{2}, \ldots x_{m}\right\}}} \frac{\partial^{m}}{\partial t_{1} \ldots \partial t_{m}} \sum_{X \in \mathcal{S}_{j}, X \supset B} \frac{1}{|X|_{j}}{ }^{f} K_{j}^{N_{2} \#}(X, 0)\right|_{t_{1}=0, \ldots t_{m}=0} \\
& =\left.\sum_{j=j_{0}}^{q-1} \sum_{\substack{B \in \mathcal{B}_{j}\left(\Lambda_{N}\right) \\
B^{*} \supset\left\{x_{1}, x_{2}, \ldots x_{m}\right\}}} \frac{\partial^{m}}{\partial t_{1} \ldots \partial t_{m}} \sum_{X \in \mathcal{S}_{j}, X \supset B} \frac{1}{|X|_{j}} f K_{j}^{N_{1} \#}(X, 0)\right|_{t_{1}=0, \ldots t_{m}=0}
\end{aligned}
$$

because for $0 \leq j \leq q-1,{ }_{f} K_{j}^{N \#}(X, 0)$ only depend on $\phi$ within $X^{*}$ and $X^{*} \subset \Lambda_{q}$ which is the center $q$-block of $\Lambda_{N_{1}} \subset \Lambda_{N_{2}}$. Therefore,

$$
\begin{aligned}
\left|{ }_{f} F_{N_{2}}-{ }_{f} F_{N_{1}}\right| & \leq\left|\sum_{j=q}^{N_{2}-1} \sum_{\substack{B \in \mathcal{B}_{j}\left(\Lambda_{N}\right) \\
B^{*} \supset\left\{x_{1}, x_{2}, \ldots x_{m}\right\}}} \frac{\partial^{m}}{\partial t_{1} \ldots \partial t_{m}} \sum_{X \in \mathcal{S}_{j}, X \supset B} \frac{1}{|X|_{j}}{ } K_{j}^{N_{2} \#}(X, 0)\right|_{t_{1}=0, \ldots t_{m}=0} \mid \\
& +\left|\sum_{j=q}^{N_{1}-1} \sum_{\substack{B \in \mathcal{B}_{j}\left(\Lambda_{N}\right) \\
B^{*} \supset\left\{x_{1}, x_{2}, \ldots x_{m}\right\}}} \frac{\partial^{m}}{\partial t_{1} \ldots \partial t_{m}} \sum_{X \in \mathcal{S}_{j}, X \supset B} \frac{1}{|X|_{j}}{ } K_{j}^{N_{1} \#}(X, 0)\right|_{t_{1}=0, \ldots t_{m}=0} \mid
\end{aligned}
$$

Then using (175) with $\mu=1 / 2$ instead of $\mu=1 / M=L^{-\eta+\epsilon}$, we obtain:

$$
\begin{gathered}
\left|\sum_{j=q}^{N_{2}-1} \sum_{\substack{B \in \mathcal{B}_{j}\left(\Lambda_{N}\right) \\
B^{*} \supset\left\{x_{1}, x_{2}, \ldots x_{m}\right\}}} \frac{\partial^{m}}{\partial t_{1} \ldots \partial t_{m}} \sum_{X \in \mathcal{S}_{j}, X \supset B} \frac{1}{|X|_{j}} f K_{j}^{N_{2} \#}(X, 0)\right|_{t_{1}=0, \ldots t_{m}=0} \mid \\
\leq 2^{d(d+1)} n_{3}\left(d, \frac{A}{2}\right) 2 \frac{m ! r 2^{-q}}{a^{m}}
\end{gathered}
$$


and

$$
\begin{gathered}
\left|\sum_{j=q}^{N_{1}-1} \sum_{\substack{B \in \mathcal{B}_{j}\left(\Lambda_{N}\right) \\
B^{*} \supset\left\{x_{1}, x_{2}, \ldots x_{m}\right\}}} \frac{\partial^{m}}{\partial t_{1} \ldots \partial t_{m}} \sum_{X \in \mathcal{S}_{j}, X \supset B} \frac{1}{|X|_{j}}{ }^{f} K_{j}^{N_{1}} \#(X, 0)\right|_{t_{1}=0, \ldots t_{m}=0} \mid \\
\leq 2^{d(d+1)} n_{3}\left(d, \frac{A}{2}\right) 2 \frac{m ! r 2^{-q}}{a^{m}}
\end{gathered}
$$

That means we have:

$$
\left|{ }_{f} F_{N_{2}}-{ }_{f} F_{N_{1}}\right| \leq 2^{d(d+1)} n_{3}\left(d, \frac{A}{2}\right) 4 \frac{m ! r 2^{-q}}{a^{m}} \rightarrow 0
$$

when $q \rightarrow \infty$.

Combining this with (158) and (167), we can conclude that $\lim _{N \rightarrow \infty}\left\langle\prod_{k=1}^{m} \partial_{\mu_{k}} \phi\left(x_{k}\right)\right\rangle^{t}$ exists.

Remark. We have $N$-uniformly boundedness on correlation functions and

$\lim _{N \rightarrow \infty} \mathcal{G}^{t}\left(x_{1}, x_{2}, \ldots x_{m}\right)$ exists. Therefore the bounds are held for infinite volume limit

\subsection{When $f(\phi)=\sum_{k=1}^{m} t_{k} \exp \left(i \partial_{\mu_{k}} \phi\left(x_{k}\right)\right)$}

Using exactly the same argument as the above subsection, we obtain these following results:

Theorem 10 For any small $\epsilon>0$, with $L, A$ sufficiently large (depending on $\epsilon$ ), let $\eta=\min \{d / 2,2\}$ we have:

$$
\left|\left\langle\prod_{k=1}^{m} \exp \left(i \partial_{\mu_{k}} \phi\left(x_{k}\right)\right)\right\rangle^{t}\right| \leq \frac{m !}{a^{m}} \operatorname{diam}^{-\eta+\epsilon}\left(x_{1}, \ldots x_{2}\right)
$$

where a depends on $\epsilon, L, A$

Theorem 11 With L, A sufficiently large, the infinite volume limit of the truncated correlation function $\lim _{N \rightarrow \infty}\left\langle\prod_{k=1}^{m} \exp \left(i \partial_{\mu_{k}} \phi\left(x_{k}\right)\right)\right\rangle^{t}$ exists

\subsection{Other cases}

We can consider $f(\phi)=\sum_{k=1}^{m} t_{k} f_{k}(\phi)\left(x_{k}\right)$ with

$* t_{k} \in \mathbb{C}$

$* x_{k} \in \mathbb{Z}^{d}$ are different points.

* $f_{k}$ is bounded in the sense that there are some $M_{k}, m_{k} \geq 0$ such that

$$
\left\|f_{k}\left(\left\{x_{k}\right\}, \phi\right)\right\|_{0} \leq M_{k}\|\phi\|_{\Phi_{0}}+m_{k}
$$

With the same argument as above cases, we have:

Theorem 12 For any small $\epsilon>0$, with $L, A$ sufficiently large (depending on $\epsilon$ ), let $\eta=\min \{d / 2,2\}$ we have:

$$
\left|\left\langle\prod_{k=1}^{m} f_{k}(\phi)\left(x_{k}\right)\right\rangle^{t}\right| \leq \frac{m !}{a^{m}} \operatorname{diam}^{-\eta+\epsilon}\left(x_{1}, \ldots x_{2}\right)
$$

where a depends on $\epsilon, L, A$

Theorem 13 With L, A sufficiently large, the infinite volume limit of the truncated correlation function $\lim _{N \rightarrow \infty}\left\langle\prod_{k=1}^{m} f_{k}(\phi)\left(x_{k}\right)\right\rangle^{t}$ exists 
In the case $f=\sum_{k=1}^{m} t_{k} W_{0}\left(\left\{x_{k}\right\}\right)$, with $W_{0}\left(\left\{x_{k}\right\}\right)=z W\left(1,\left\{x_{k}\right\}\right)$ as in (42). Using the Lemma 2 (or the lemma 4 in [5] ), these $W_{0}\left(\left\{x_{k}\right\}\right)$ satisfy those above conditions. The $W_{0}\left(\left\{x_{k}\right\}\right)$ are actually the density of the dipoles at $x_{k}$ used in [2]. Applying theorems 12 and 13, we obtain these results:

Corollary 1 For any small $\epsilon>0$, with $L, A$ sufficiently large (depending on $\epsilon$ ), let $\eta=\min \{d / 2,2\}$ we have:

$$
\left|\left\langle\prod_{k=1}^{m} W_{0}\left(\left\{x_{k}\right\}\right)\right\rangle^{t}\right| \leq \frac{m !}{a^{m}} \operatorname{diam}^{-\eta+\epsilon}\left(x_{1}, \ldots x_{2}\right)
$$

This result somehow looks like the theorem (1.1.2) in 2]. However it gives estimates for truncated correlation functions of $(p \geq 2)$ points instead of some estimate for only 2 points.

Corollary 2 With L, A sufficiently large, the infinite volume limit of the truncated correlation function $\lim _{N \rightarrow \infty}\left\langle\prod_{k=1}^{m} W_{0}\left(\left\{x_{k}\right\}\right)\right\rangle^{t}$ exists

Remark. We can consider the more general form $f(\phi)=\sum_{k=1}^{m} t_{k} f_{k}(\phi)$ with

$* t_{k} \in \mathbb{C}$

* $A_{k} \equiv \operatorname{supp} f_{k}$ are pairwise disjoint and $\left|A_{k}\right|<\infty$

* $f_{k}$ is bounded in the sense that there are some $M_{k}, m_{k} \geq 0$ such that

$$
\left\|f_{k}\left(A_{k}, \phi\right)\right\|_{0} \leq M_{k}\|\phi\|_{\Phi_{0}}+m_{k}
$$

Then we still get similar results as in Theorems 12 and 13 .

\section{A Kac-Siegert Transformation}

By expanding the exponential in (6) and carrying out the Gaussian integrals, we can rewrite ${ }_{0} Z_{N}$ as

$$
\begin{aligned}
{ }_{0} Z_{N} & =\int\left(\sum_{n \geq 0} \frac{z^{n}}{n !} \prod_{i=1}^{n} \sum_{x_{i} \in \Lambda_{N} \cap \mathbb{Z}^{d}} \int_{\mathbb{S}^{d-1}} d p_{i}\left(e^{i p_{i} \cdot \partial \phi\left(x_{i}\right)}+e^{-i p_{i} \cdot \partial \phi\left(x_{i}\right)}\right) / 2\right) d \mu_{C}(\phi) \\
& =\int\left(\sum_{n \geq 0} \frac{z^{n}}{n !} \prod_{i=1}^{n} \sum_{x_{i} \in \Lambda_{N} \cap \mathbb{Z}^{d}} \int_{\mathbb{S}^{d-1}} d p_{i} e^{i p_{i} \cdot \partial \phi\left(x_{i}\right)}\right) d \mu_{C}(\phi) \\
& =\sum_{n \geq 0} \frac{z^{n}}{n !} \prod_{i=1}^{n} \sum_{x_{i} \in \Lambda_{N} \cap \mathbb{Z}^{d}} \int_{\mathbb{S}^{d-1}} d p_{i} \int e^{i \sum_{k=1}^{n} p_{k} \cdot \partial \phi\left(x_{k}\right)} d \mu_{C}(\phi) \\
& =\sum_{n \geq 0} \frac{z^{n}}{n !} \prod_{i=1}^{n} \sum_{x_{i} \in \Lambda_{N} \cap \mathbb{Z}^{d}} \int_{\mathbb{S}^{d-1}} d p_{i} \exp \left(\frac{-1}{2} \sum_{1 \leq k, j \leq n}\left(p_{k} \cdot \partial\right)\left(p_{j} \cdot \partial\right) C\left(x_{k}, x_{j}\right)\right)
\end{aligned}
$$

which is exactly the same as the grand canonical partition function (5).

\section{Acknowledgments}

This work was in partial fulfillment of the requirements for the Ph.D. degree at the University at Buffalo, State University of New York. The author owes deep gratitude to his Ph.D. advisor Jonathan D. Dimock for his continuing help and support. Dimock's prior investigations of infinite volume limit for the dipole gas [5] have served as a framework for much of the current work. 


\section{References}

[1] D.C.Brydges, Lectures on the renormalisation group, in Statistical Mechanics, IAS/Park City Math. Ser., volume 16 (American Mathematical Society, Providence, RI, 2009), pp. 7-93

[2] D. Brydges and G Keller, Correlation functions of general observables in dipole-type systems, Helv. Phys. Acta 67, 43-116 (1994).

[3] D.C.Brydges, G. Guadagni, P.K. Mitter, Finite range decomposition of Gaussian processes, J. Stat. Phys. 115, 415-449, (2004).

[4] D.C. Brydges, H.T. Yau., Grad $\phi$ perturbations of massless Gaussian fields, Commun. Math. Phys. 129, 351-392, (1990).

[5] J. Dimock, Infinite volume limit for the dipole gas, Journal of Statistical Physics, 35, 393-427, (2009).

[6] J. Dimock, Quantum mechanics and quantum field theory. A mathematical primer., Cambridge University Press, Cambridge, 2011.

[7] J.Dimock, T.R. Hurd. A renormalization group analysis of correlation functions for the dipole gas, J. Stat. Phys. 66, 1277-1318, (1992).

[8] J. Dimock, T. Hurd, Sine-Gordon revisited, Ann. Henri Poincaré 1, 499-541 (2000).

[9] P. Falco, Kosterlitz-Thouless transition line for the two dimensional Coulomb gas, Comm. Math. Phys. 312, no. 2, 559-609 (2012)

[10] J. Frohlich, Y.M. Park, Correlation inequalities and the thermodynamic limit for classical and quantum continuous systems, Commun. Math. Phys. 59, 235-266 (1978)

[11] J. Frohlich, T. Spencer, On the statistical mechanics of classical Coulomb and dipole gases, J. Stat. Phys. 24, 617-701 (1981)

[12] K. Gawedzki and A. Kupiainen, Lattice dipole gas and $(\nabla \phi)^{4}$ models at long distance, decay of correlations and scaling limit, Commun. Math. Phys. 92, 531 (1984).

[13] M. Kac, On the partition function of one dimensional gas, Phys. Fluids, vol. 2, 8 (1959).

[14] M. Reed, B. Simon, Methods of modern mathematical physics, Vol IV, Academic Press (1978).

[15] B. Simon, Functional integration and quantum physics, Academic Press (1979)

[16] A. F. J. Siegert, Partition functions as averages of functionals of Gaussian random functions, Physica, Vol. 26, 530-535, (1960)

[17] G. Zhao, Dipole-Dipole Correlations for the sine-Gordon Model, arXiv:1108.3232 